Ratan Joarder, Gregor Christoffer Gebel, Thomas Mosbach, Two-dimensional numerical simulation of a decaying laser spark in air with radiation loss, International Journal of Heat and Mass Transfer 63 (2013) 284-300

The original publication is available at http://www.elsevier.com/

http://dx.doi.org/10.1016/j.ijheatmasstransfer.2013.03.072 


\title{
Two-dimensional numerical simulation \\ of a decaying laser spark in air with radiation loss
}

\author{
R. Joarder ${ }^{1)}$, G. C. Gebel, T. Mosbach \\ Institute of Combustion Technology, German Aerospace Center (DLR), 70569 Stuttgart, \\ Germany
}

\begin{abstract}
Results are presented on two-dimensional numerical simulation of a decaying laser spark after the air breakdown. Radiation loss is modeled in a finite volume framework for a finite wavelength range of the emitted rays. The redistribution of assumed absorbed energy among different parts, namely the blast wave energy, the radiative heat energy and the leftover energy is quantified. About 5\% of the absorbed energy is found to be trapped around the breakdown region after the blast wave moves away. A prediction is made about the actual available energy for ignition of a combustible mixture. A distinct rollup motion around the breakdown region is demonstrated from the simulation, which is in accordance with other numerical and experimental observations. Finally, the evolution of the blast wave radius with time is validated against in-house experimental data.
\end{abstract}

\section{Introduction}

When a laser pulse is focused in air, a part of the pulse energy is absorbed. For sufficiently high absorbed energy air breakdown takes place, which is initiated by multiphoton ionization followed by electron cascade breakdown [1, 2]. Upon cessation of the pulse, the breakdown zone undergoes changes which involve electromagnetic radiation, chemical and fluiddynamic phenomena. Electromagnetic radiation is emitted from the breakdown zone primarily by free electrons undergoing transitions to other free states or to bound states of atoms and ions, and also by bound electrons as they undergo transitions to other bound states. Chemical changes involve short term recombination or dissociation of the species present depending on the temperature and pressure of the breakdown zone. Due to the pressure gradient between the breakdown zone and the ambience, velocity is induced in the initially stagnant air and depending on the absorbed energy, some fluid dynamic phenomena are observed, namely blast wave, vortex flow etc. These processes could grossly be called as the

\footnotetext{
${ }^{1}$ Corresponding author, e-mail : Ratan.Joarder@dlr.de
} 
decay of the spark. Finally, there are no gradients in pressure, temperature and velocity around the breakdown zone.

After the independent discovery of laser-induced breakdown by Marker (1963) and Meyerland and Haught (1963), several possible uses of it came up [3]. The blast wave emanating from the laser induced breakdown zone could produce propulsive power to small vehicles. The energy deposited by a laser could reduce wave drag on blunt bodies. Laser sparks could also be used to ignite combustible mixtures. It has certain advantages over conventional spark ignition. Those are (a) greater control over timing and location of the spark due to its non-intrusive nature, (b) higher efficiency and lower $\mathrm{NO}_{\mathrm{x}}$ emissions in the ignition of lean combustible mixtures by employing multiple ignition points. Also, (c) a continuous laser spark could work as a source of radicals, which would work as flame holders. This concept could replace traditional flame holders in supercritical but low Mach number flows because of reduced drag.

As mentioned above, one of the possible uses of a laser spark is the ignition of combustible mixtures. Several studies are carried out to understand the laser-induced ignition process [48]. Those involve numerical simulations of both air breakdown chemistry and the fluid dynamics of the resulting blast wave following the breakdown [5], numerical simulations of only the gas dynamic effects following the breakdown assuming initial thermodynamic equilibrium $[4,6]$, comparison of computed and experimental density, pressure, temperature, and velocity outside the laser spot [7] and investigations of fluid dynamic effects including the roll up of the plasma core following laser induced breakdown by solving the NavierStokes equations in Fourier space [8]. In the authors' knowledge, the total radiation loss during the decay process is not described anywhere including the above references. Experimental determination of total radiation loss of a decaying laser spark is indeed a difficult task. In this paper an attempt is made for the above numerically. Also, the variations of the internal energy, species mass concentration, and temperature with time and space around the breakdown region, which are important to understand the ignition process by a laser spark, is also described in the present study. Additionally, the distribution of the total absorbed energy into its constituent parts namely the blast wave energy, the radiation energy and the left-over energy are quantified. Here the blast wave energy refers to the kinetic energy of the gas behind the blast wave. The left-over energy refers to the energy left in the system after the pressure behind the blast wave has nearly equilibrated itself with the ambience. 
This paper is organized as follows. Sections II and III describe the simulation methodology, followed by results in section IV. A brief description about the in-house experiments and comparison of blast wave radius from numerical simulation and experiments is given in section V. The distribution of total energy among different part is described in section VI. A short summary in section VII concludes the paper.

\section{Numerical Simulation}

In simplified engineering approach, the Navier-Stokes equations are solved to determine the thermodynamic (e.g. pressure, temperature) and flow variables (e.g. velocity) in fluid flows. However, additional equations may be required to be solved depending on the physical situation of the problem concerned and depending on required detail of the flow-field.

In the present numerical simulation, the plasma is assumed to reach LTE (Local Thermodynamic Equilibrium) and chemical equilibrium conditions on termination of the laser pulse. The numerical simulation tries to find the resulting thermodynamic, fluid dynamic and chemical situations for about $1 \mathrm{~ms}$ subsequent to the laser induced plasma formation. A laser-induced micro-plasma reaches LTE within nanoseconds after termination of the laser pulse. This is verified experimentally [9] and this assumption is used in previous numerical studies as well [6]. The main parameter which determines whether a laser induced micro-plasma reaches LTE or not is determined by minimum electron number density, so that the collisional processes dominate with respect to the radiative processes. It is found that for the kind of absorbed energy $(93 \mathrm{~mJ}$ ) used in this study, the plasma reaches LTE. It is important to note that this local equilibrium may apply only to a certain subset of particles in the system. In a radiating gas, the photons being emitted and absorbed by the gas need not be in thermodynamic equilibrium with each other or with the other gas particles of the gas in order for LTE to exist. During the decay of the laser spark, chemical non-equilibrium may arise and this is taken care of by solving additional species conservation equation with finite rate reaction chemistry.

The initial shape of a laser spark after cessation of the laser pulse is like a tear drop [8, 10]. The axial symmetry of a laser spark allows one to carry out the simulation in two dimensions. The solution domain can be assumed to be a slice (plane) passing through the axis of a laser 
spark. The Navier-Stokes equations along with species conservation equations in vector integral form for a two-dimensional case may be expressed as

$$
\frac{\partial}{\partial t} \int_{V} U d v+\oint_{S}(H-F) \cdot \vec{\kappa} d s=\int_{V} S d v
$$

where $U$ and $S$ are the column vectors containing conservative variables and source terms respectively, $H$ and $F$ are second order flux tensors containing inviscid and viscous fluxes respectively, and $\vec{k}$ is the outward normal to $d s$. The expressions for $U, S, H, F$ and the associated variables are given in the appendix.

The inviscid fluxes are discretized by the approximate Riemann solver of Roe [11]. Roe's scheme gives approximate values of the cell normal inviscid fluxes as

$$
H_{n}\left(U_{L}, U_{R}\right)=\frac{1}{2}\left(H_{n}\left(U_{L}\right)+H_{n}\left(U_{R}\right)\right)-\frac{1}{2} \sum_{i=1}^{3+N} \tilde{\alpha}_{i}\left|\tilde{\lambda}_{i}\right| \tilde{K}^{i}
$$

where $H_{n}\left(U_{L}\right), H_{n}\left(U_{R}\right), \tilde{\alpha}_{i}, \tilde{\lambda}_{i}, \tilde{K}^{i}$ are the normal fluxes at the left and right side of cell interface (control surface), Roe average wave strengths, eigenvalues and eigenvectors respectively of the matrix $\vec{A} \cdot \vec{\kappa}$ (details can be found in the appendix). The presence of additional variables in chemical non-equilibrium case makes the determination of Roe average quantities from the equations originally given by Roe under determined. In the present simulations, the average derivative terms are determined from the method described by Shuen et al. [12]. Roe's scheme results in unphysical expansion shock without correction to sonic rarefaction waves. In the present study the approach given by Toro [13] is followed to fix the problem. The original Roe's scheme is first order accurate in space. To increase the spatial accuracy to third order in space in smooth parts of the solution, the MUSCL extrapolation strategy [14] is pursued along with the Van Albada limiter [15] which suppresses spurious oscillations near a strong discontinuity.

The calculation of viscous fluxes requires the value of velocity and temperature derivative at cell interfaces. Here the procedure described by Peyret and Taylor [16] is followed.

The vector of source terms (S) contains non-zero values from $4^{\text {th }}$ to $(3+\mathrm{N})^{\text {th }}$ element, where $\mathrm{N}$ is the number of species. The $4^{\text {th }}$ non-zero term is the divergence of radiation heat flux with a negative sign $-\nabla \cdot q^{\text {rad }}$ and the other non-zero terms represent species generation rates per unit volume. 
There are several methods to calculate the heat flux due to radiation. Among those the zone method, the Monte Carlo method, flux methods and hybrid methods are popular. The finite volume method $[17,18]$ is one of the flux methods. The finite volume method in radiation heat transfer can share the same computational grid and philosophy, which is used for solving the fluid flow problem in a finite volume formulation. Hence, it eliminates the need to interpolate temperature, absorption coefficient, and average radiation intensity during the solution process. In the present simulations, the finite volume method is used to solve the radiative transfer equation (RTE) due to the above mentioned convenience. The heat flux due to radiation is calculated once the radiation intensity field is known from the solution of RTE. The RTE has the form

$$
\frac{d I_{v}(r, \hat{s})}{d s}=-\left(\kappa_{a v}+\sigma_{s v}\right) I_{v}(r, \hat{s})+\kappa_{a v} I_{b v}(r)+\sigma_{s v} \bar{I}_{v}(r, \hat{s})
$$

where $I_{v}(r, \hat{s})$ is the spectral intensity of radiation with frequency $v$ at a spatial location $r$ over the path length $d s$ in the direction of unit vector $\hat{s}, \kappa_{a v}$ and $\sigma_{s v}$ are the absorption and scattering coefficients respectively. The spectrally integrated intensity is given by

$$
I(r, \hat{s})=\int_{0}^{\infty} I_{v}(r, \hat{s}) d v
$$

The strong spectral dependency of radiative properties of radiating gases render the simple grey gas approximation unacceptable if the radiation heat loss is to be calculated quantitatively. However it is also very difficult to integrate the above equation in the whole range because of requirements of high computational power and lack of data of absorption and other coefficients. One of the procedures for handling the frequency dependence of the coefficients in the RTE is the multi-group method [19] which assigns a given photon to one of the $G$ frequency groups. All photons within a given group are treated in the same way, assigning average properties, such as the absorption coefficient, to that group.

$$
\begin{array}{r}
\kappa_{a v}(r, v, T)=\kappa_{k}(r, T), v_{k} \leq v \leq v_{k+1}, k=1,2, . ., G \\
I(r, \hat{s})=\sum_{k=1}^{G} I_{k}(r, \hat{s}), I_{k}(r, \hat{s})=\int_{v_{k}}^{v_{k+1}} I_{v}(r, \hat{s}) d v
\end{array}
$$

Then the RTE could be written as

$$
\frac{d I_{k}(r, \hat{s})}{d s}=\kappa_{a k}\left(b_{k} \frac{\sigma T^{4}}{\pi}-I_{k}(r, \hat{s})\right)
$$


ignoring the scattering coefficients. Here $b_{k}$ is the fraction of $\frac{\sigma T^{4}}{\pi}$ lying within the $\mathrm{k}^{\text {th }}$ group i.e.

$$
b_{k}=\frac{\int_{v_{k}}^{v_{k+1}} B(v) d v}{\int_{0}^{\infty} B(v) d v}=\frac{\int_{v_{k}}^{v_{k+1}} B(v) d v}{\frac{\sigma T^{4}}{\pi}}
$$

$B(v)$ is the Planck's function given by

$$
B_{v}(v, T)=\frac{2 h v^{3}}{c^{2}\left[\exp \left(\frac{h v}{k T}\right)-1\right]}
$$

where $\mathrm{h}=6.6256 \times 10^{-34} \mathrm{Js}$ is the universal Planck's constant, $\mathrm{k}=1.3804 \times 10^{-23} \mathrm{~J} / \mathrm{K}$ is the Boltzmann constant, $\mathrm{c}=3 \times 10^{8} \mathrm{~m} / \mathrm{s}$ is the speed of light in vacuum and $\mathrm{T}$ is the absolute gas temperature. In [19] the frequency range considered is $10^{13}-6 \times 10^{15} \mathrm{~s}^{-1}$ which is divided into 12 non-uniform frequency groups. Finally, the divergence of radiation flux is given by

$$
\begin{gathered}
\nabla \cdot q^{r a d}=\sum_{k=1}^{G} \kappa_{a k}\left(\pi b_{k} \frac{\sigma T^{4}}{\pi}-G_{k}\right) \\
G_{k}=\sum_{l} I_{k}^{l} \Delta \Omega^{l}
\end{gathered}
$$

Because radiation is an inherently 3-dimensional phenomenon, radiation must be considered for the entire solid angle $4 \pi$ over a two-dimensional computational domain. In the present simulation, first the radiation heat flux is calculated for a solid angle $2 \pi$ and then multiplied by two to find the total radiation heat flux. The procedure for calculating total radiation heat loss is explained in section VI.

The expressions for other source terms in the vector $\mathrm{S}$ is given in the appendix. The source terms in the species conservation equations are treated implicitly in time following Bussings and Murman [20]. The inviscid fluxes, the viscous fluxes and the radiation terms are treated explicitly in time. The update formula for the conservative variables in a single time stage $\Delta \mathrm{t}$ $=\mathrm{t}^{\mathrm{n}+1}-\mathrm{t}^{\mathrm{n}}$ is given by

$$
U^{n+1}=U^{n}-\frac{\Delta t}{\Delta V}\left[\sum_{i=1}^{n_{s}}\left(H_{i s}^{n}-F_{i s}^{n}\right) \kappa_{i} \Delta s_{i}\right]+\frac{S^{n} \Delta t}{I-\Delta t\left(\frac{\partial S}{\partial U}\right)^{n}}+S^{1 n} \Delta t
$$

where $S$ is the vector of source terms containing non-zero elements only for the species conservation equations and $S^{1 n}$ is the vector of source terms containing non-zero elements 
only for the energy equation due to the divergence of the radiation heat flux. For any computational cell it is convenient to write the above equation as

$$
\frac{\partial U}{\partial t}=R(U)
$$

The time integration of the above equation to steady state is carried out based on a multistage Runge-Kutta method [21] which comprises of the sequence

$$
\begin{aligned}
& U^{(0)}=U^{n} \\
& U^{(k)}=U^{(0)}+\alpha_{k} R\left(U^{(k-1)}, \Delta t\right), k=1, . ., m \\
& U^{(n+1)}=U^{m}
\end{aligned}
$$

where $\mathrm{m}$ denotes the number of stage. Values of $\alpha_{k}$ for a six stage third order accurate integration are $\left[\begin{array}{llllll}0.0742 & 0.1393 & 0.2198 & 0.3302 & 0.5181 & 1.000\end{array}\right]$. The time step for any computational cell $(\mathrm{i}, \mathrm{j})$ is prescribed in [21] so that it satisfies the linear stability criterion.

\section{Initial and boundary conditions, domain size and solution methodology}

Initially the laser spark is of trapezoidal shape [4]. The dimension of the trapezoid is given in FIG. 1. In this region the gas is at chemical equilibrium. The equilibrium composition is calculated by Gibbs free energy minimization procedure [22] and assuming that the internal energy per unit volume of the gas to be $\left(93 \mathrm{~mJ} / 3 \mathrm{~mm}^{3}\right)$. Here $93 \mathrm{~mJ}$ is the absorbed energy (obtained from experiments) and $3 \mathrm{~mm}^{3}$ is the assumed focal volume i.e. the volume into which the laser is focused. The calculation also determines the temperature and pressure for the assumed values of internal energy and volume. This temperature is assigned at the rightmost point of the trapezoid on the axis of symmetry along $\mathrm{X}$ direction. Temperature of the other points on this axis is determined assuming an exponential distribution. Temperature perpendicular to the axis of symmetry (along $\mathrm{Y}$ direction) is determined following normal distribution with the maximums on the axis of symmetry.

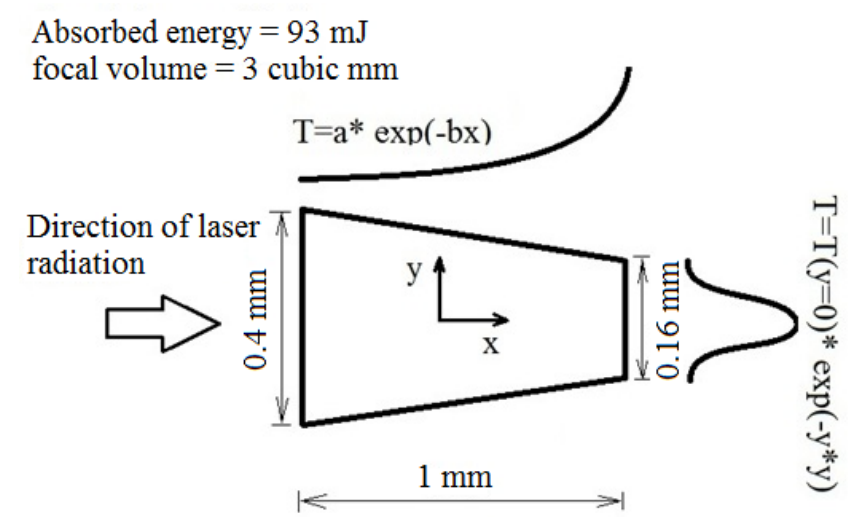


FIG. 1. The shape and temperature distribution of a breakdown zone after the laser pulse [4].

A square domain of $20 \times 20 \mathrm{~mm}^{2}$ dimensions is chosen for the numerical simulation. The domain is discretized by $500 \times 500$ square control volumes. The number of cells is chosen after few simulations, when it is observed that there are no changes in the solution nature and almost no changes in the values of dependent variables if the numbers of control volumes are increased. The angular space of $2 \pi$ is discretized by 2 and 8 control angles in the polar and azimuthal directions respectively.

In the finite rate chemistry model five species, namely $\mathrm{O} 2, \mathrm{O}, \mathrm{N} 2, \mathrm{~N}, \mathrm{NO}$ and eleven elementary reaction steps [23] are considered for dissociation and recombination of air. The thermodynamic and transport properties of the species are assumed to be temperature dependent [24, 25]. Then the assumed profile of temperature e.g. exponential along the direction of laser irradiation and Gaussian in the perpendicular direction is determined and fed into the central part of the domain. For flows with finite-rate reaction chemistry, the species concentration $\mathrm{C}_{\mathrm{i}}$ depends not only on transport of fluid, but also on the progress of chemical reactions; therefore, a priori determination of an EOS (Equation of State) is not possible and the EOS has to be constructed along with the solution process. If the inter molecular forces and the volume occupied the molecules are negligible, pressure p can be expressed as

$$
p=R_{u} T \sum_{i=1}^{N} \frac{C_{i}}{W_{i}}
$$

The use of ideal gas equation of state instead of Van der Waals equation is a simplification. Rest of the domain is assumed to be at a temperature and pressure of $300 \mathrm{~K}$ and 1 bar respectively. The spectral radiation intensity and the divergence of radiation heat flux are calculated from this temperature distribution. After the first iteration, the temperature is recovered using the equation

$$
\rho e=\sum_{i=1}^{N} \frac{C_{i}}{W_{i}}\left(\int_{T_{R}}^{T} C_{p i} d T+h_{f i}^{0}\right)-p
$$

and the process is repeated. The variables of equations 15 and 16 are described in the appendix.

The RTE is solved iteratively by the finite volume method [17]. In the beginning, the value of radiation intensity $I_{P}^{l}$ at the cell center of a generic cell or finite volume is determined by the equation 


$$
I_{P}^{l}=\frac{\Delta y D_{c x}^{l} I_{W}^{l}+\Delta x D_{c y}^{l} I_{S}^{l}+\kappa_{a k} b_{k} I_{b} \Delta x \Delta y \Delta \Omega^{l}}{\Delta y D_{c x}^{l}+\Delta x D_{c y}^{l}+\kappa_{a k} \Delta x \Delta y \Delta \Omega^{l}}
$$

where $\Delta y, D_{c x}^{l}, \Delta x, D_{c y}^{l}, \Delta \Omega^{l}$ are geometrical factors [17], $I_{b}$ is the thermal radiation intensity, $I_{W}^{l}, I_{S}^{l}$ are the values of radiation intensity at the cell centers of western and southern cells of $I_{P}^{l}$ respectively. The others factors are defined in section II. The solution process for radiation intensity in the RTE starts with an initial guess of radiation intensity. The value of $I_{b}$ is calculated from the initial temperature distribution. At the boundaries, in general, the leaving intensity is a summation of emitted and reflected intensities (assuming a wall which is grey-diffuse). In the present simulations, there are no walls surrounding the domain. So the boundary intensities are extrapolated from interior of the domain. This process is repeated in subsequent time steps after the temperature is recovered using equation 16. The solution is deemed converged when it satisfies the following condition:

$$
\frac{\left|I_{P}^{l}-I_{P}^{0^{0}}\right|}{I_{P}^{l}} \leq 10^{-6}
$$

Here $I_{P}^{I^{0}}$ is $I_{P}^{l}$ from the previous iteration. The values of the conservative variables at the boundaries are also extrapolated from interior of the domain. 


\section{Results}

It is observed from the numerical solutions that there are two distinct phases of the decay process of a laser spark. In the initial phase, which lasts for about $0.4 \mu \mathrm{s}$, there are rapid changes in the spatial distribution of the physical variables. The breakdown zone changes its shape from tear-drop to nearly circular. In the second phase, the changes become regular and continue till the temperature and pressure decay close to the ambient values. The distinct features of this phase are (a) separation of blast wave from the expanding hot region, (b) trapping of $\approx 5 \%$ of the absorbed energy around the central part of the domain (calculations are in section V), (c) reduction in the temperature decay rate compared to the initial period. The blast wave separation from the hot region causes a drastic reduction in the energy convection rate. So radiation and conduction are the main mechanisms of heat energy transfer during this period. The above observations are based on the following plots.

For a better comprehension of the evolution of a laser spark, the data obtained from the two-dimensional numerical simulation is rotated about the axis of symmetry by $180^{\circ}$ and is presented in FIG. 2 which shows the iso-surfaces of Mach number $(\mathrm{M}=0.4)$ of the gas behind the blast wave during the initial phase of the decay process. The contour plots presented in two-dimensions in FIG. 3-6 are the plots on a plane passing through the axis of symmetry. The values of the dependent variables e.g. pressure, temperature etc. are normalized in the contour plots by the equation $\mathrm{V}^{+}=\left(\mathrm{V}-\mathrm{V}_{\min }\right) / \mathrm{V}_{\max }$ where $\mathrm{V}^{+}$is the normalized value of $\mathrm{V}$, $\mathrm{V}_{\min }$ and $\mathrm{V}_{\max }$ are the minimum and maximum values of $\mathrm{V}$ at a certain time instant within the two-dimensional domain.

$0.00019 \mu s$

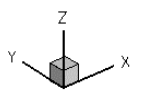

$0.069 \mu s$
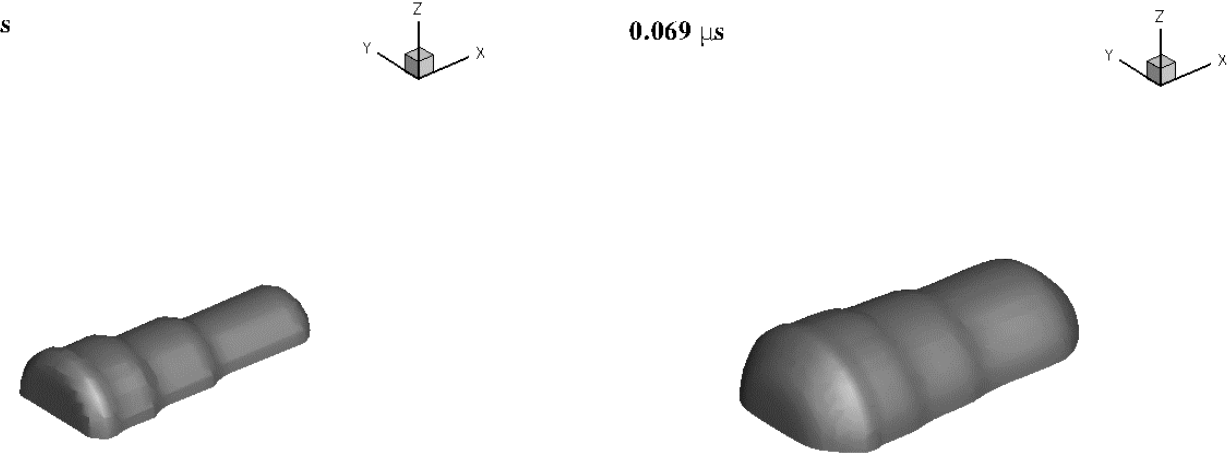
$0.35 \mu s$

$1^{2}$

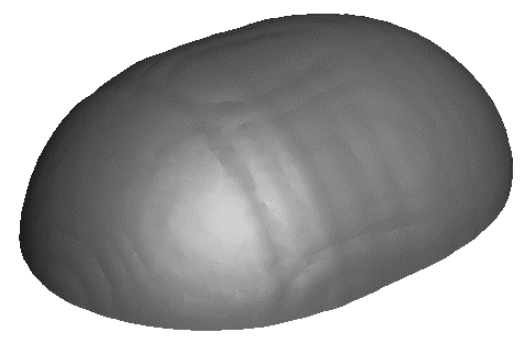

FIG. 2. Iso-surface of Mach number $(\mathrm{M}=0.4)$ during the initial phase of the decay process.
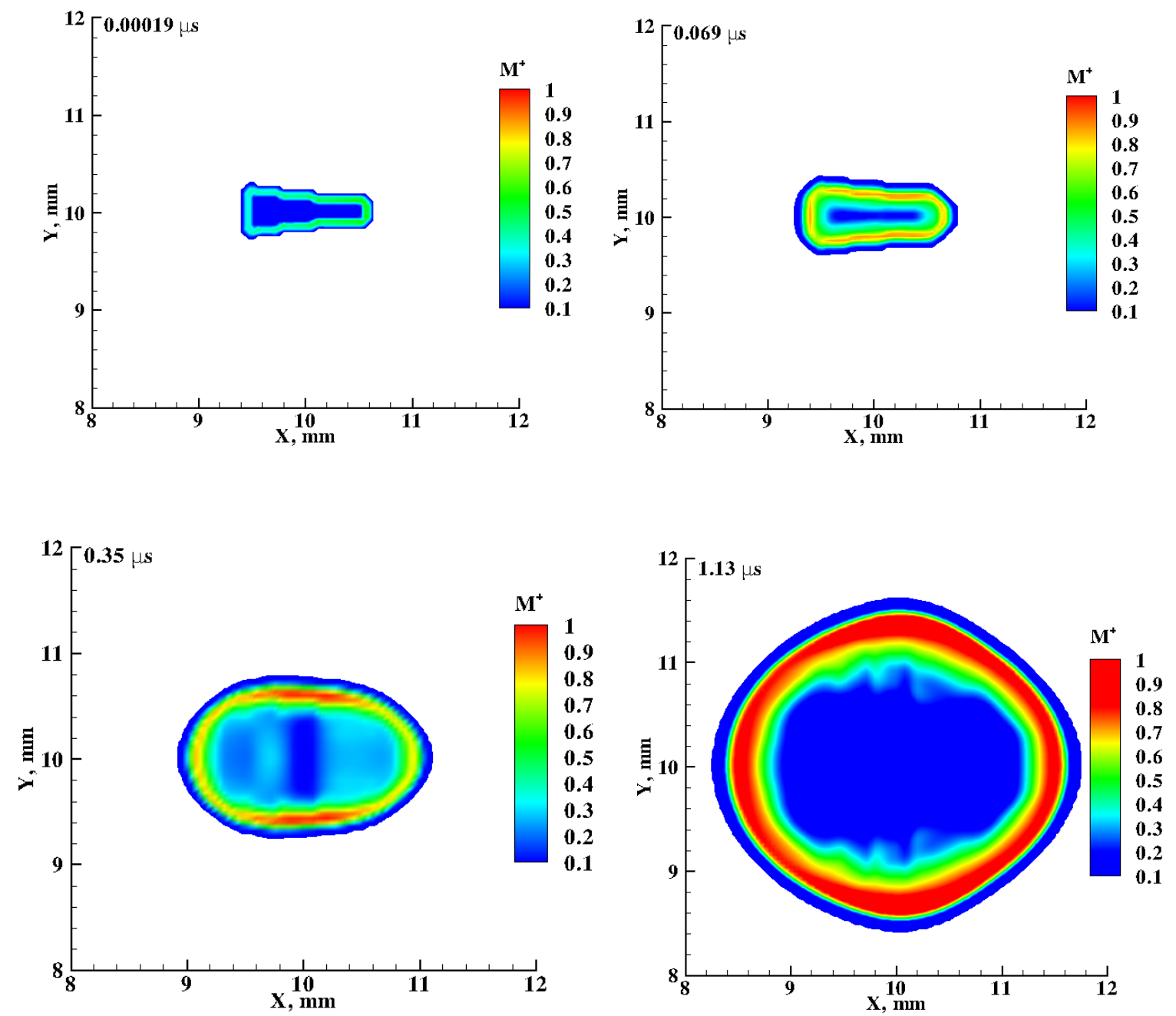

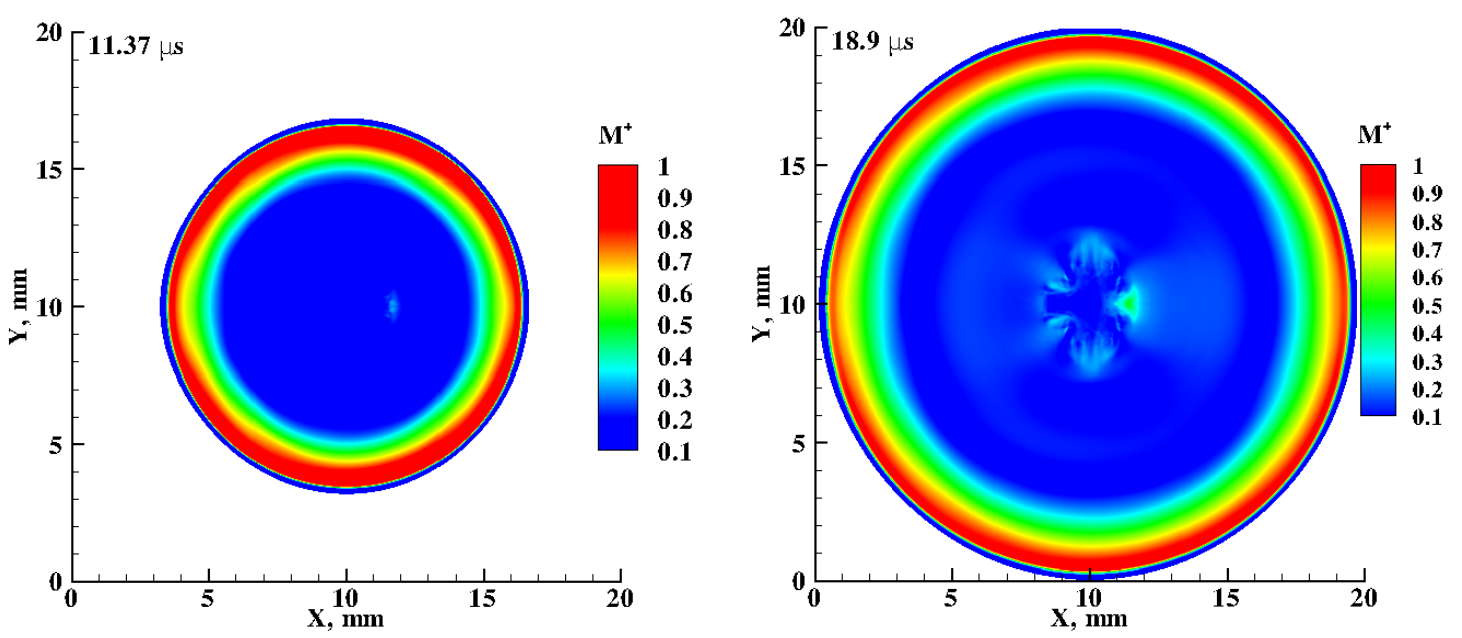

FIG. 3. Contour plots of Mach number (normalized) during the decay process.
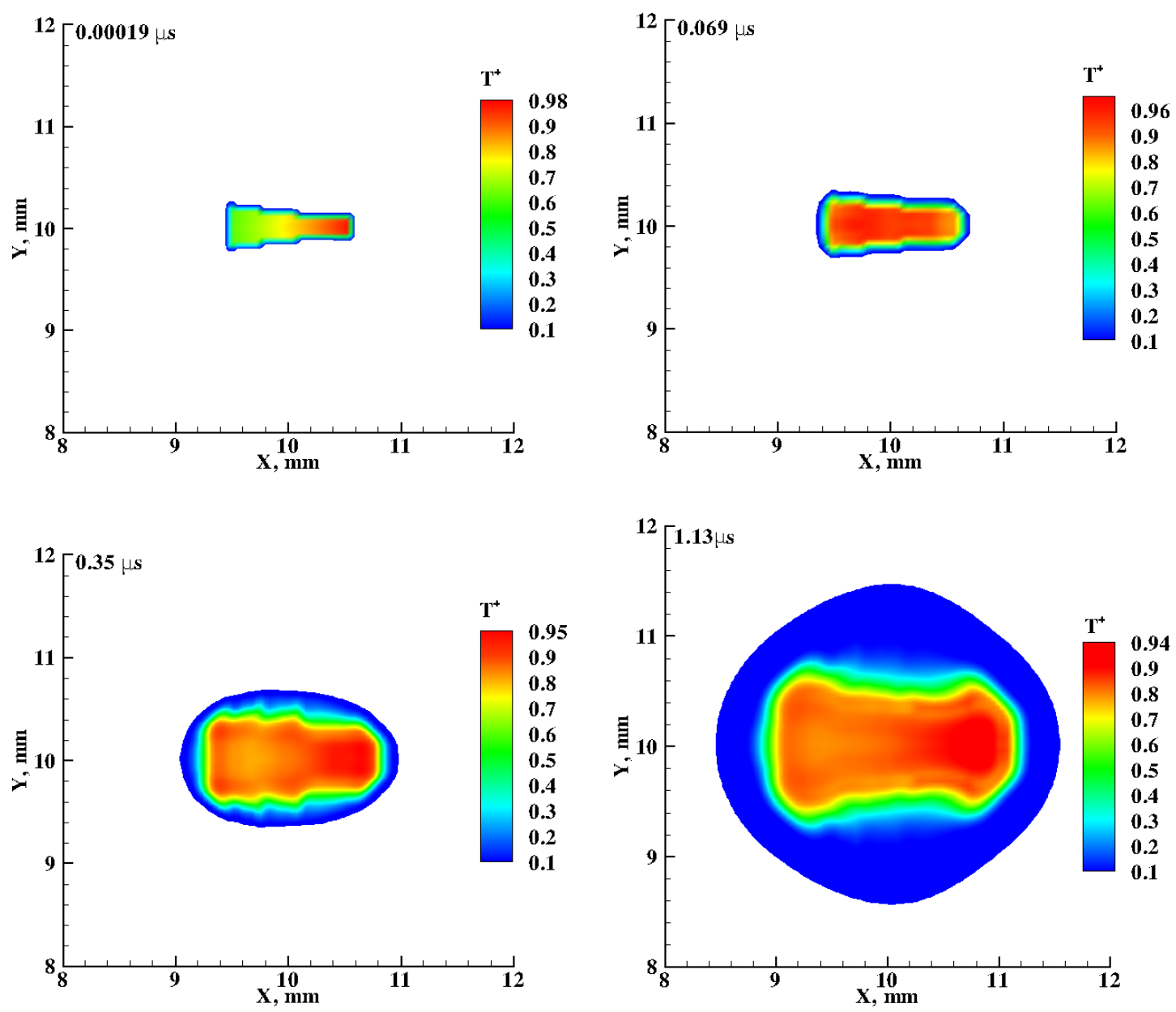

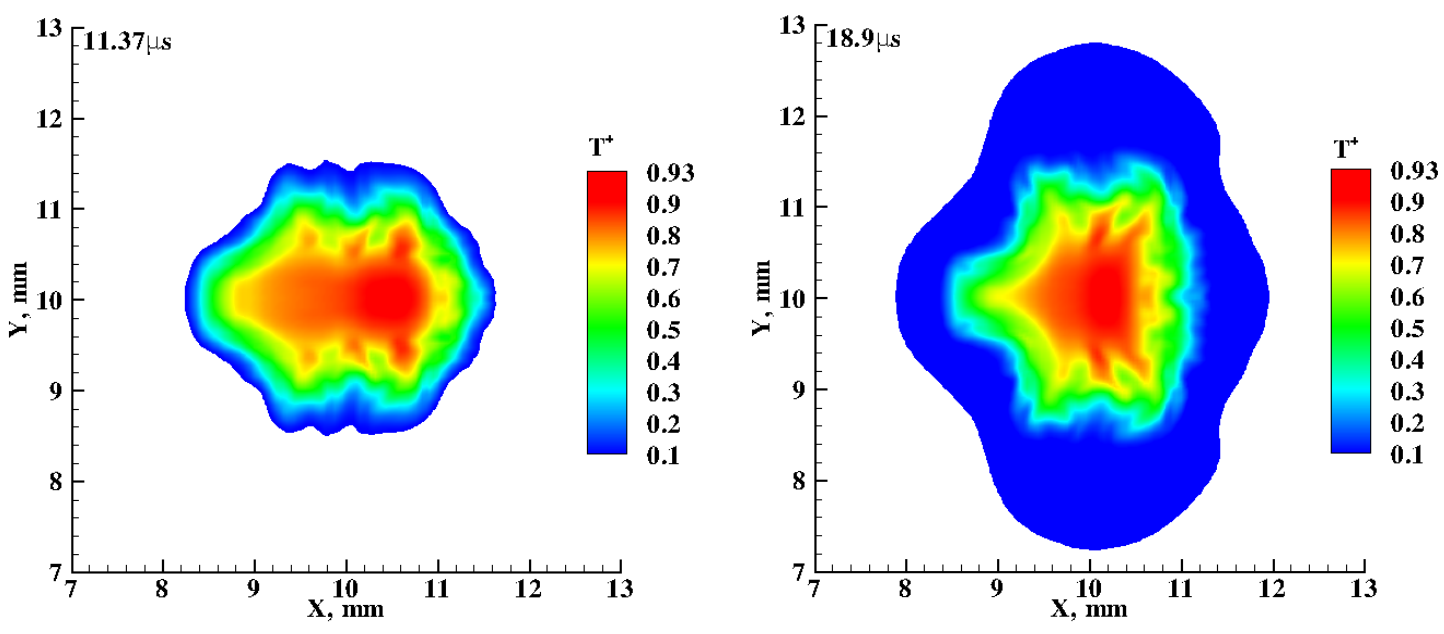

FIG. 4. Contour plots of temperature (normalized) during the decay process.
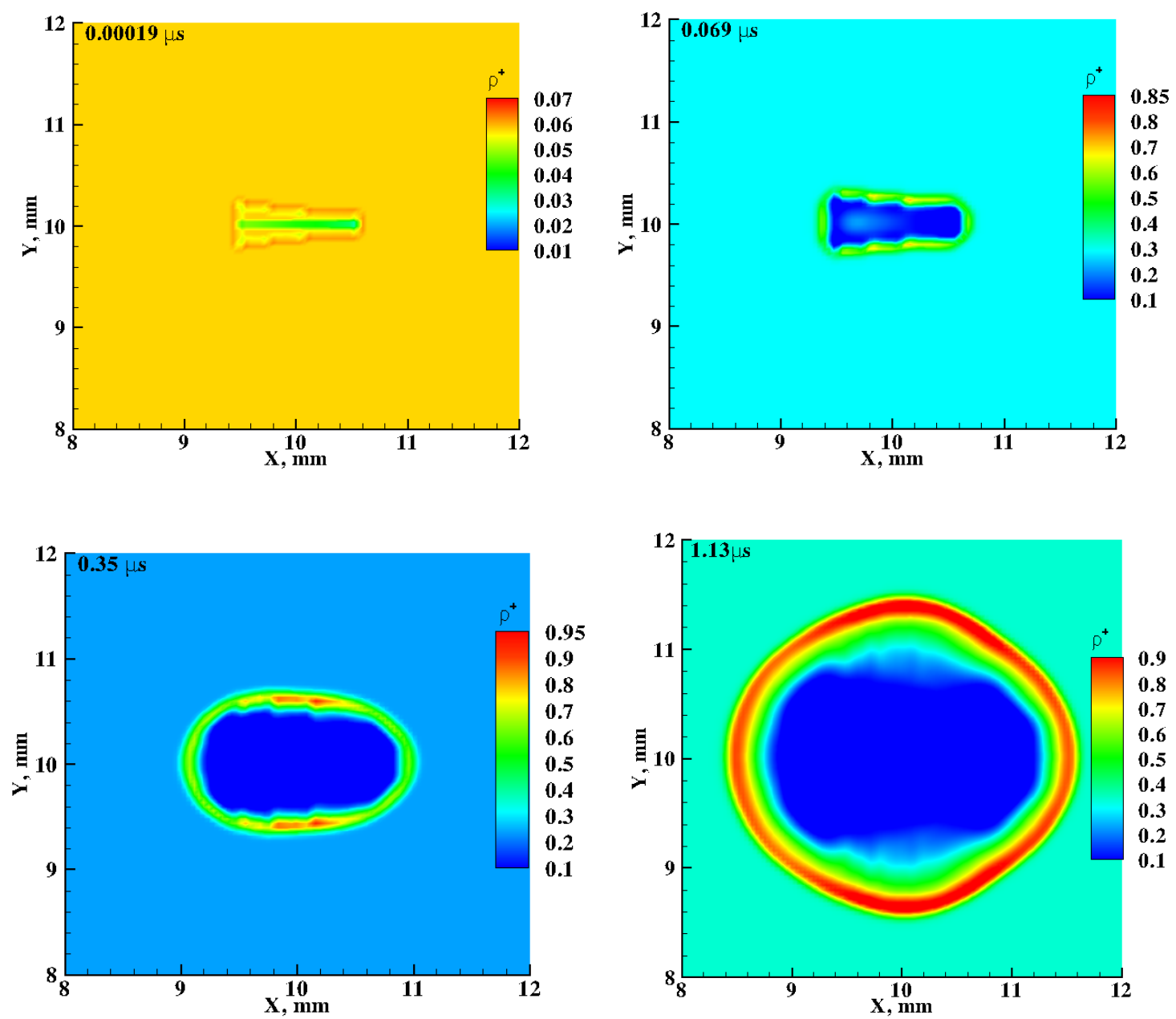

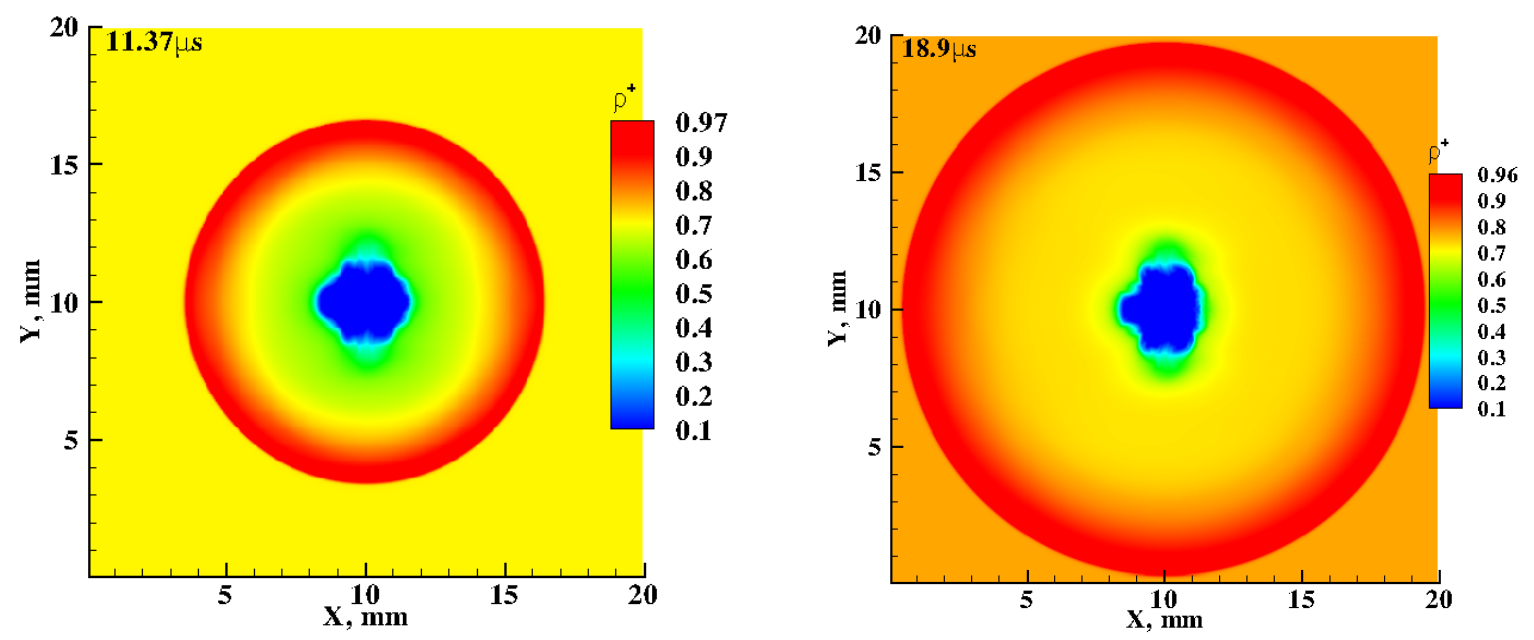

FIG. 5. Contour plots of density (normalized) during the decay process.
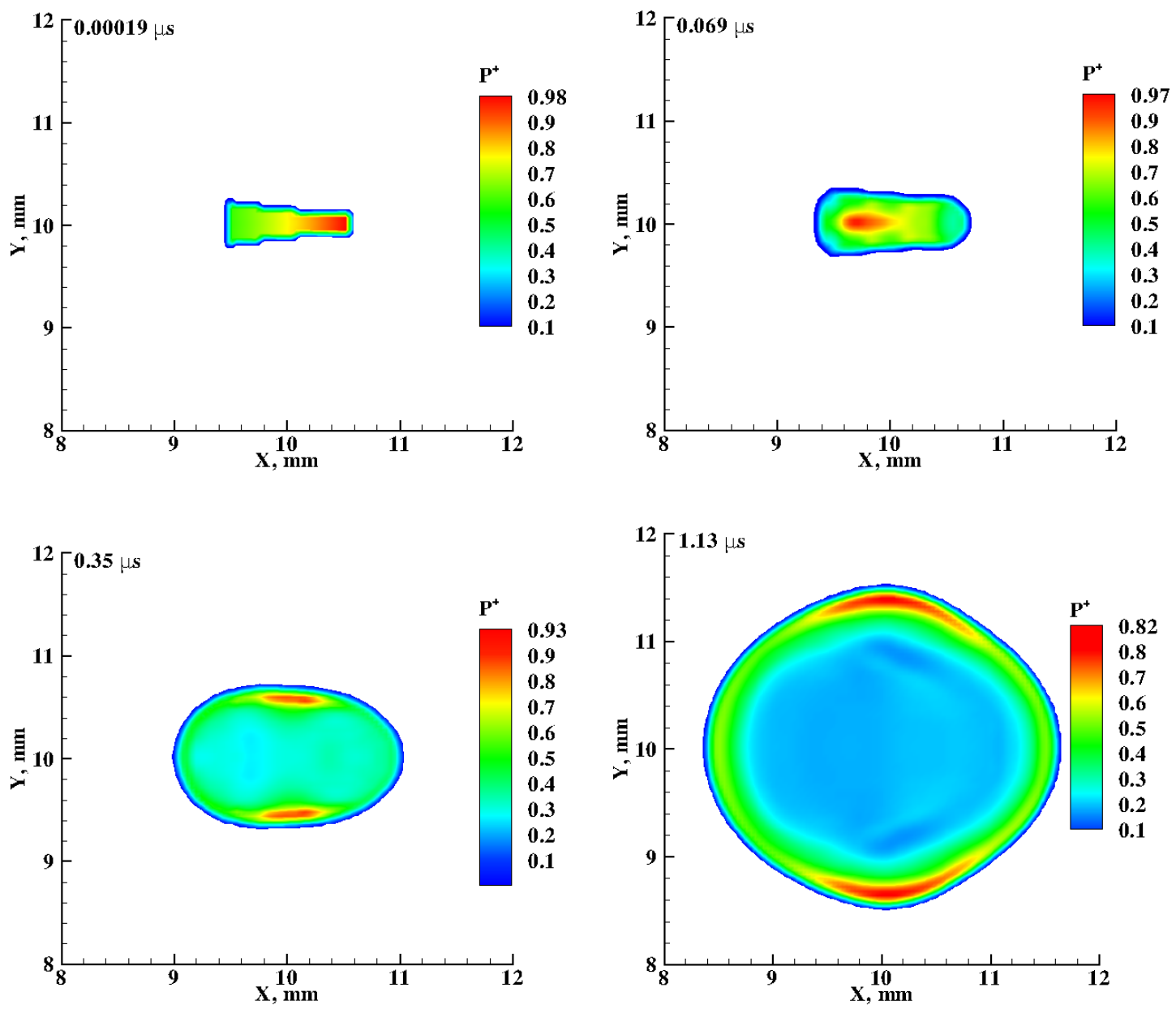

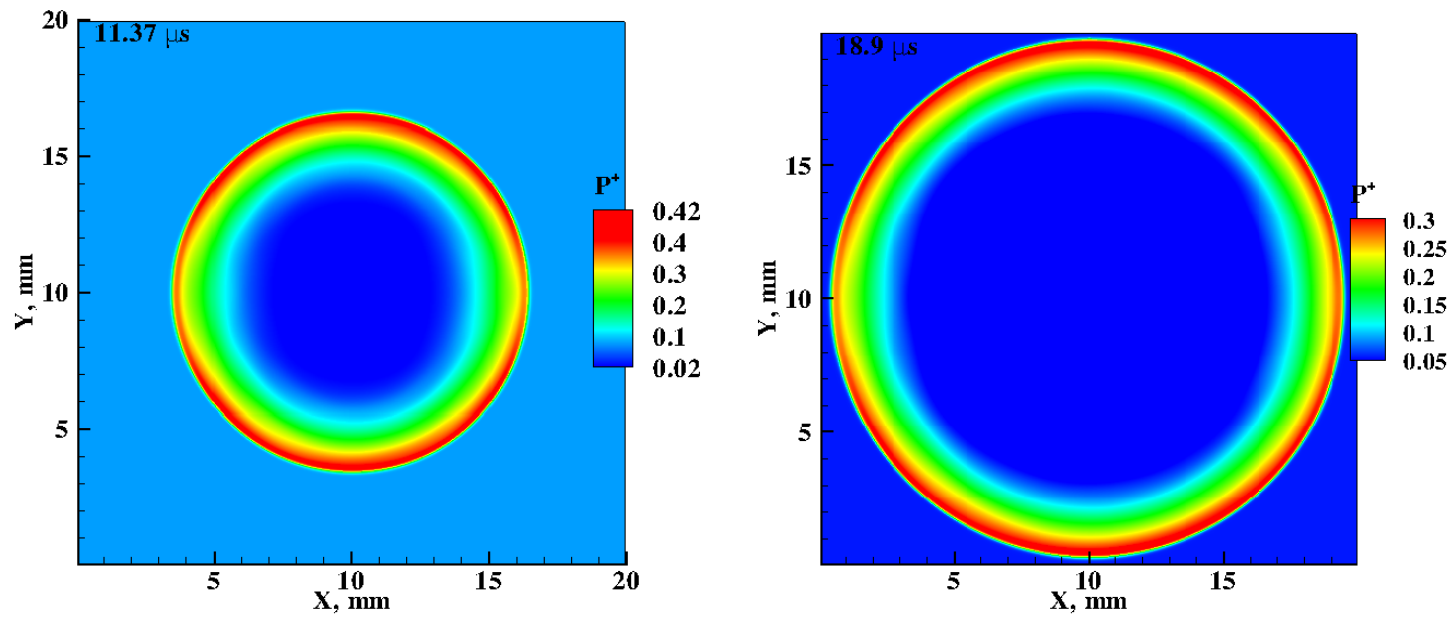

FIG. 6. Contour plots of pressure (normalized) during the decay process.
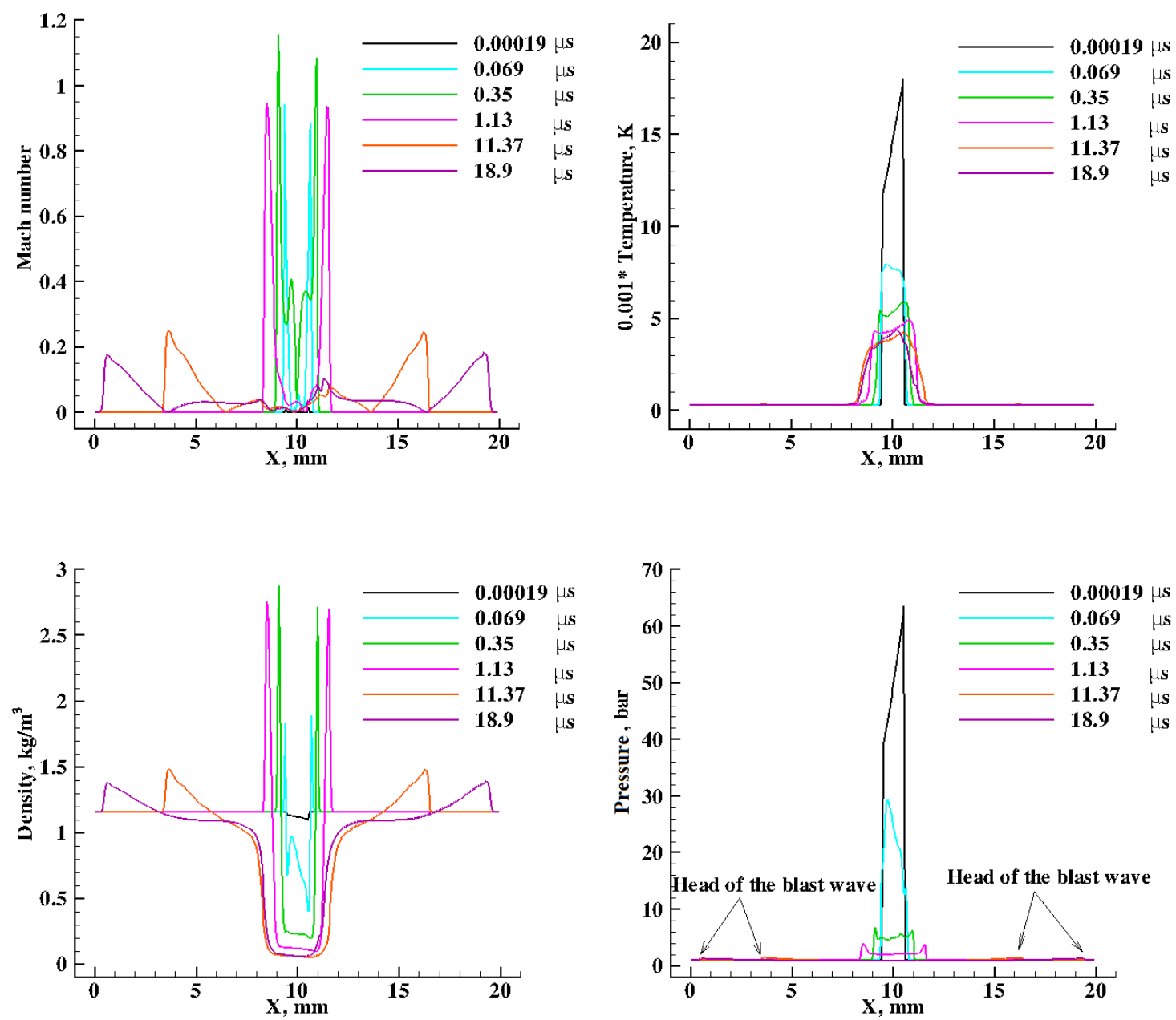

FIG. 7. Line plots of Mach number, temperature, density and pressure on a line passing through the middle of the domain along $\mathrm{x}$ axis during the decay process. 
In FIG. 7, the values of selected physical variable are plotted on a line passing through the middle of the domain along $\mathrm{x}$ axis during the decay process. Of particular interest is the temperature profile, which does not move outward as the profile of other variables e.g. pressure, density etc. In other words, the temperature change is found to be confined within 3 $\mathrm{mm}$ around the center although the pressure and density change beyond that region. It is found that in the region of $3 \mathrm{~mm}$ around the center, the dissociated species and hence, a part of the absorbed energy is trapped for time much longer than the time required for the pressure to equilibrate with the ambience. Because the blast wave has propagated away from the region, the heat is transferred mainly by radiation and thermal conduction.

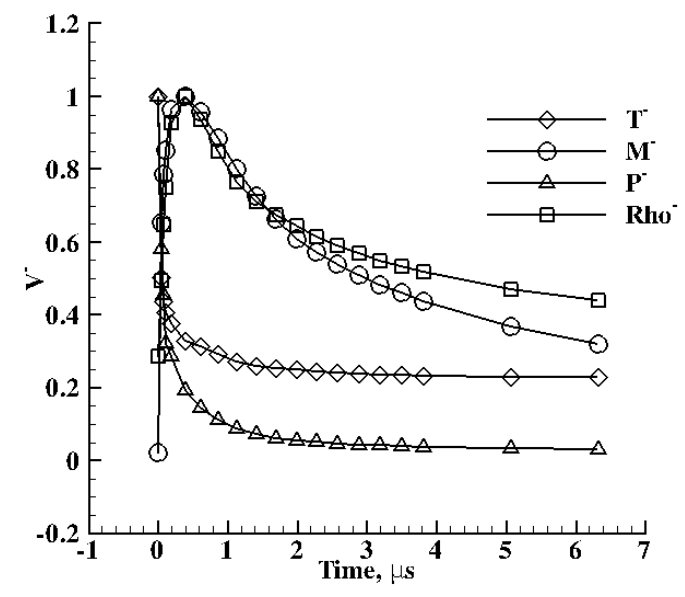

(a)

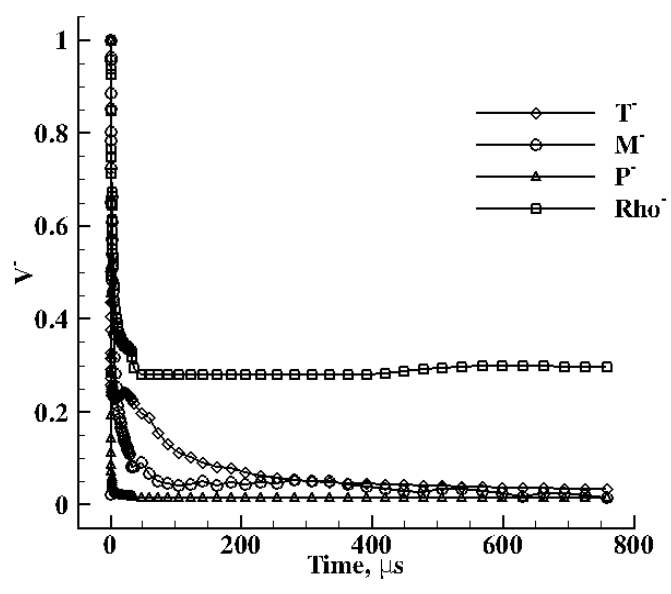

(c)

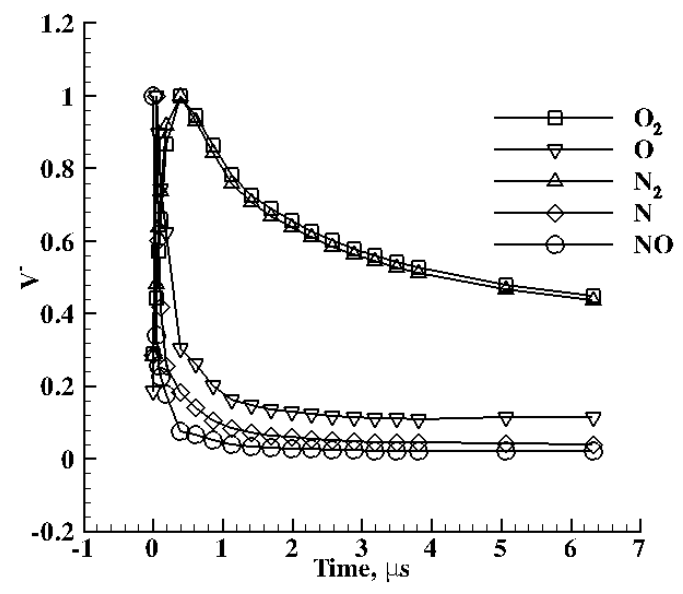

(b)

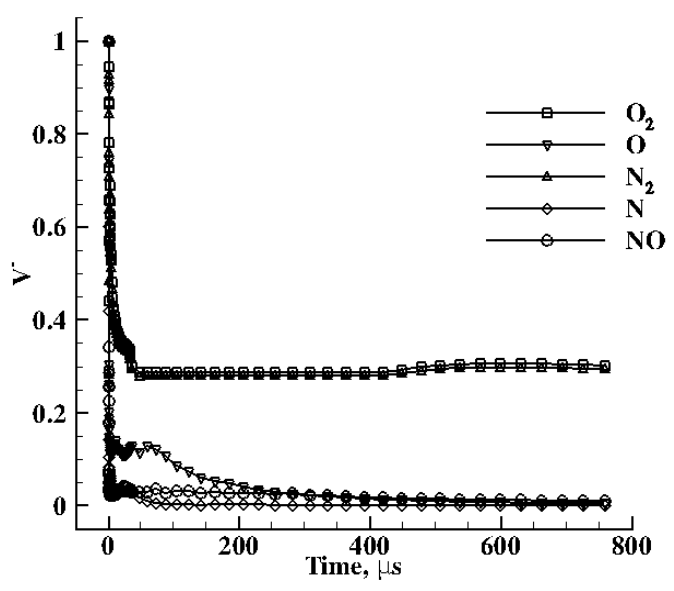

(d)

FIG. 8. The variation of the maximum value of different physical variables with time within the entire domain, (a-b) during early stages and (c-d) during the entire decay process. 
FIG. 8 shows the variations of the maximum values of different physical variable within the entire domain with time. Here, $\mathrm{V}^{-}=\mathrm{V} / \mathrm{V}_{\max }, \mathrm{V}_{\max }$ is the maximum value of $\mathrm{V}$ within the entire simulation time. For ease of understanding, four plots are given. The first two plots correspond to shorter duration during which rapid changes in the values of the physical variables occur. The last two correspond to the entire simulation period, at the end of which the values of the physical variables become approximately equal to ambient values.

FIG. 9 shows the streamlines around the central part of the domain at different instants of time. The streamlines show interesting features. Those involve horizontal line source, double point source, single point source, vertical line source (FIG. 9(a-d) respectively) during the initial phase of the decay process. At later times distinct roll up motion are observed (FIG 9(ip)). The increase in surface area due to the roll-up motion and flow field turbulence could enhance the rate of flame propagation if a laser spark is used for igniting a combustible gas mixture [2]. The flow field generated by a laser spark during the initial phase is extremely complex in nature in the sense that the density and pressure fields are irregular and change fast in time. Hence it is difficult to pin-point the reasons behind the observed patterns in the streamlines. A general explanation may be that the velocity vector follows the pressure gradient and density at any time instant and spatial location. At a later phase, the changes are much slower and predictable. The pressure in the central part goes little below one atmosphere while the pressure behind the blast wave is little above one atmosphere. Hence the streamlines change its direction from radially outward to radially inward and roll up motion are observed, which turns into a toroidal ring vortex. It is reported [8] that at short times vorticity is generated by baroclinic means while, at longer times reverse flow in the plasma core generates vorticiy. These roll up motions eventually die down, and at the end are marked by sporadic distribution (FIG. 9(r)). Similar fluid motions are also observed experimentally [26-27]. 


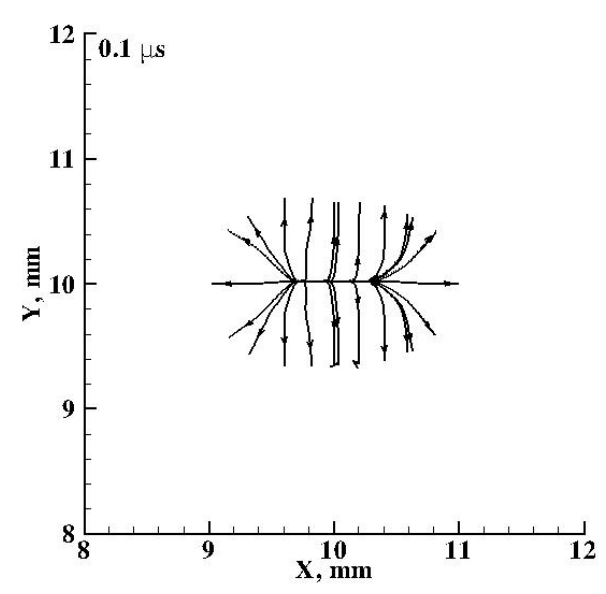

(a)

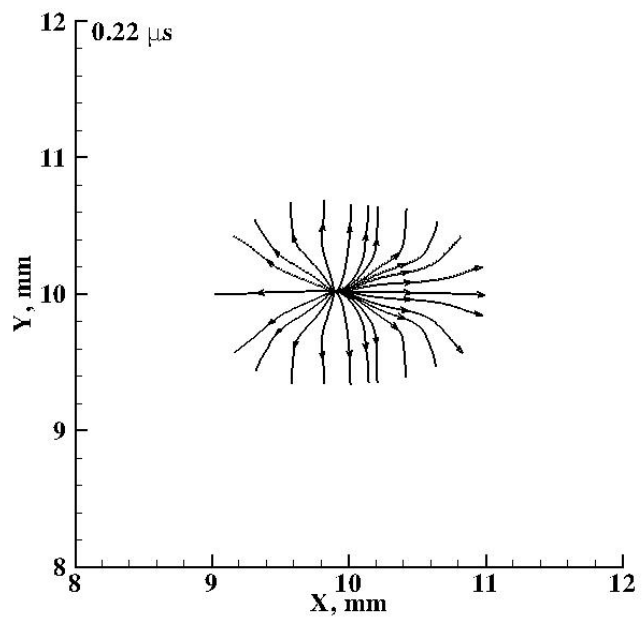

(c)

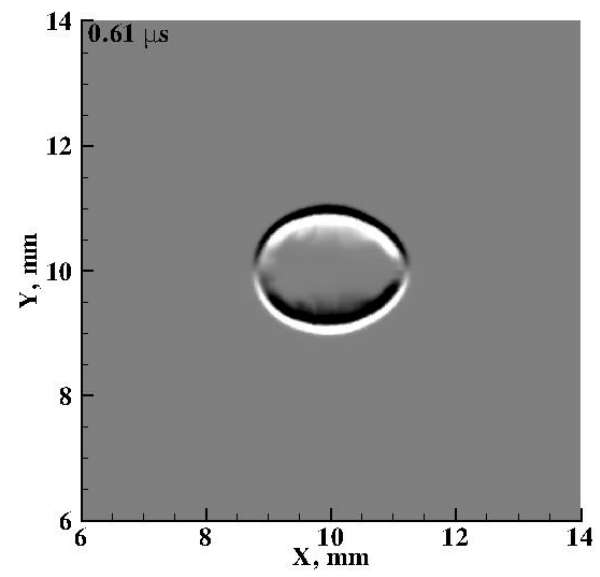

(e)

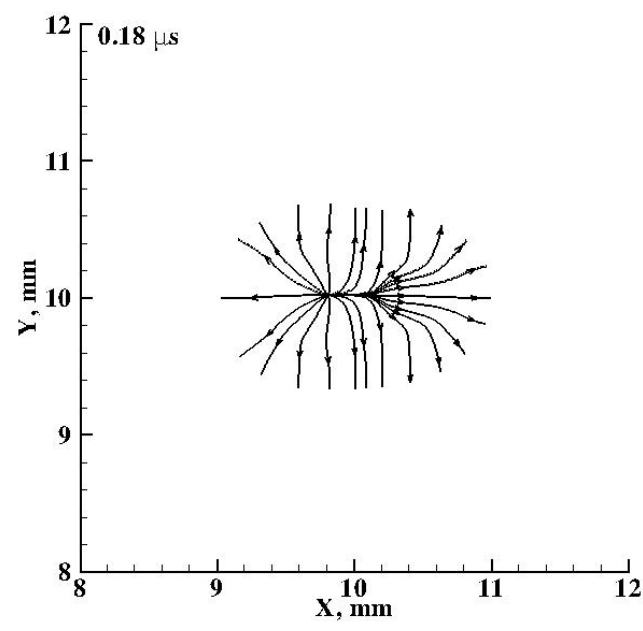

(b)

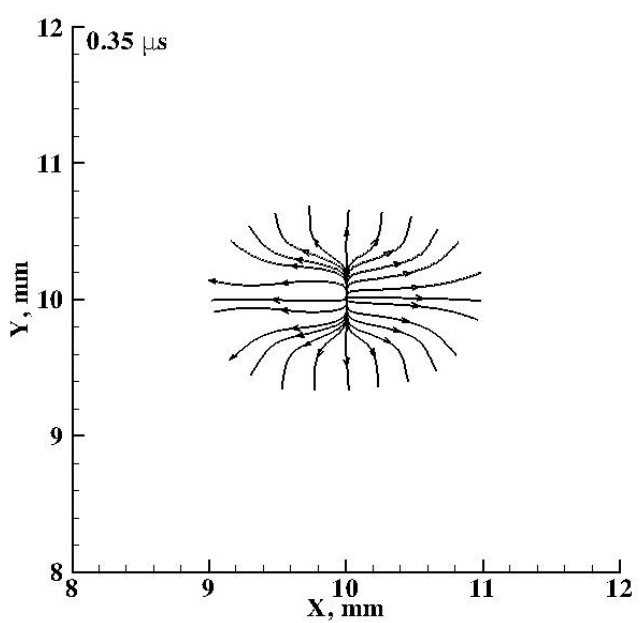

(d)

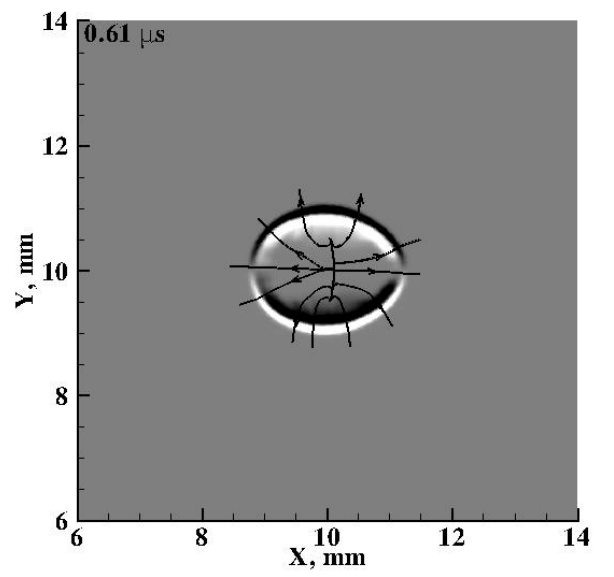

(f) 


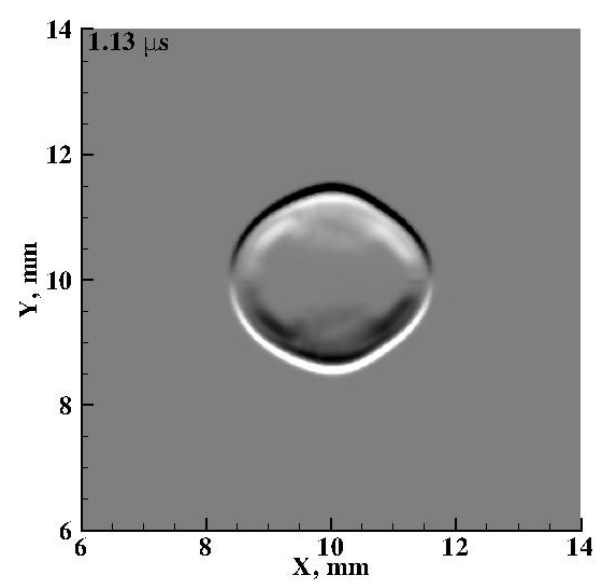

(g)

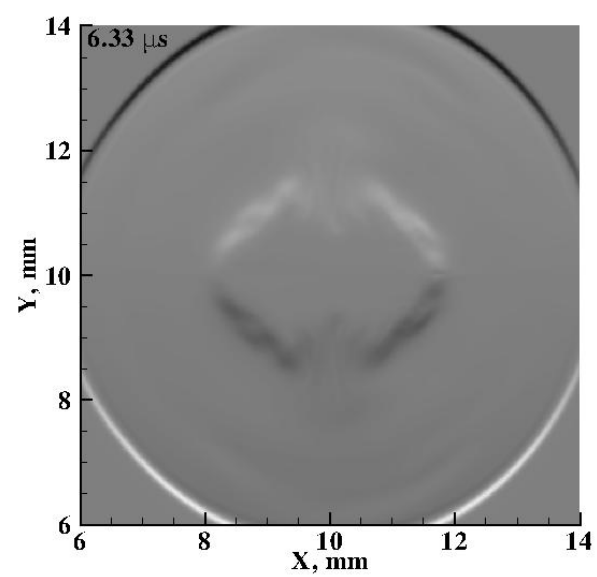

(i)

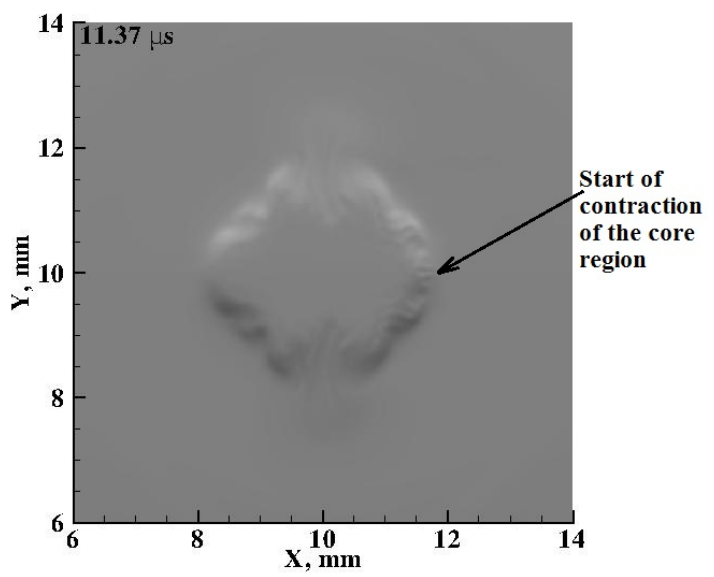

(k)

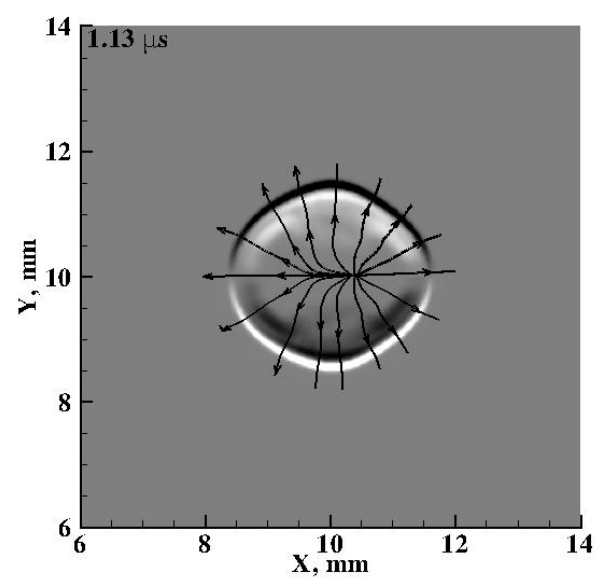

(h)

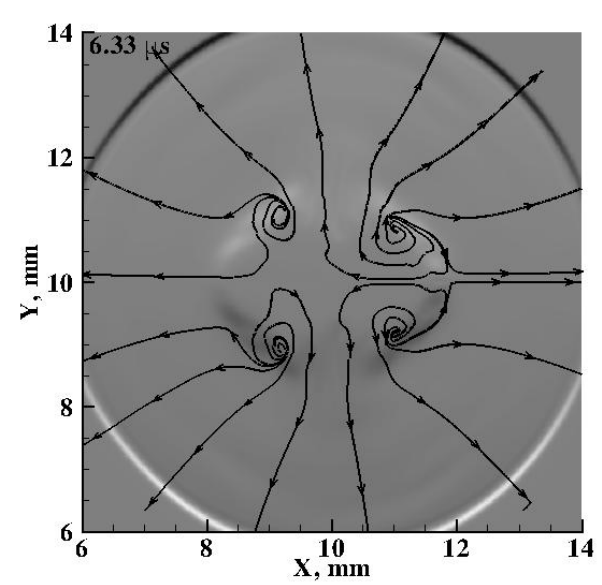

(j)

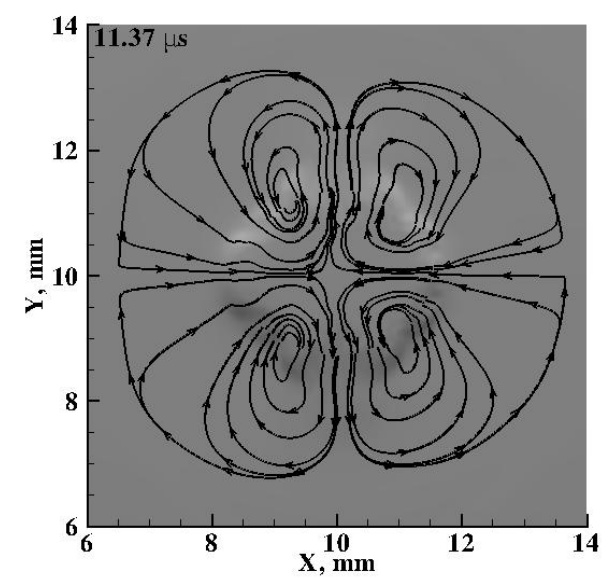

(l) 


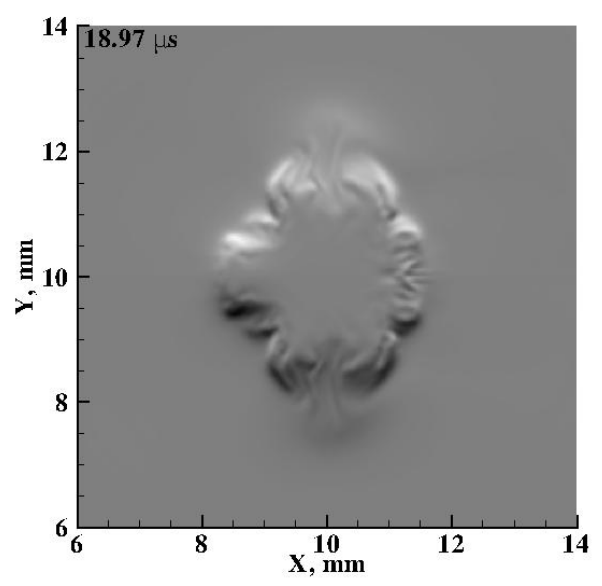

(m)

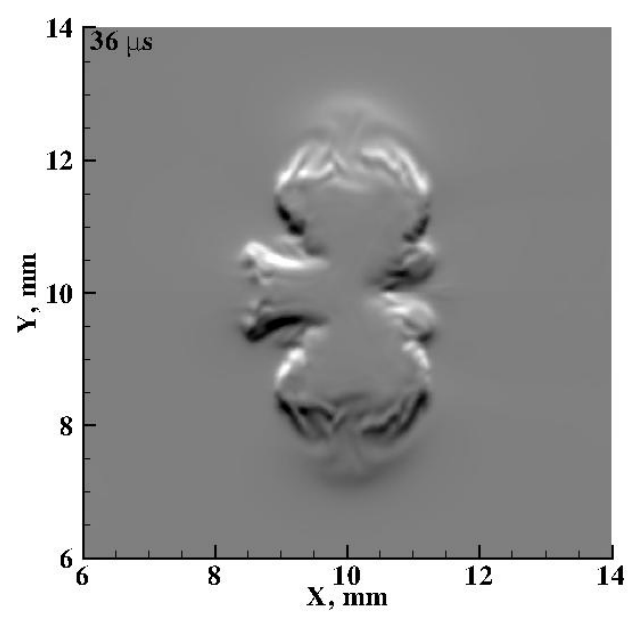

(o)

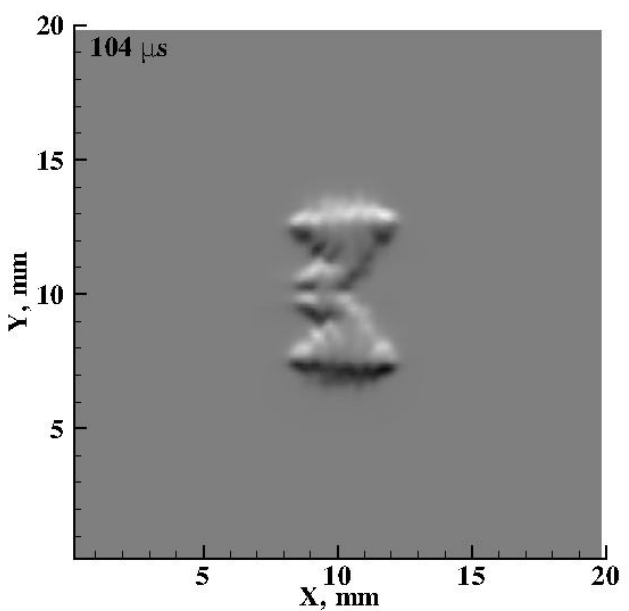

(q)

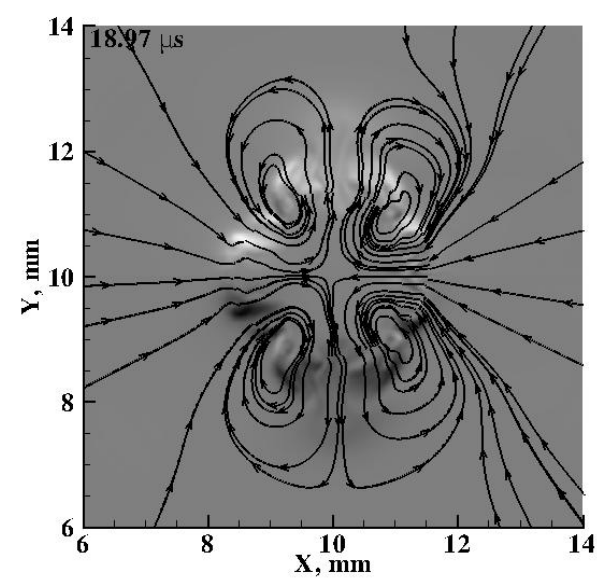

(n)

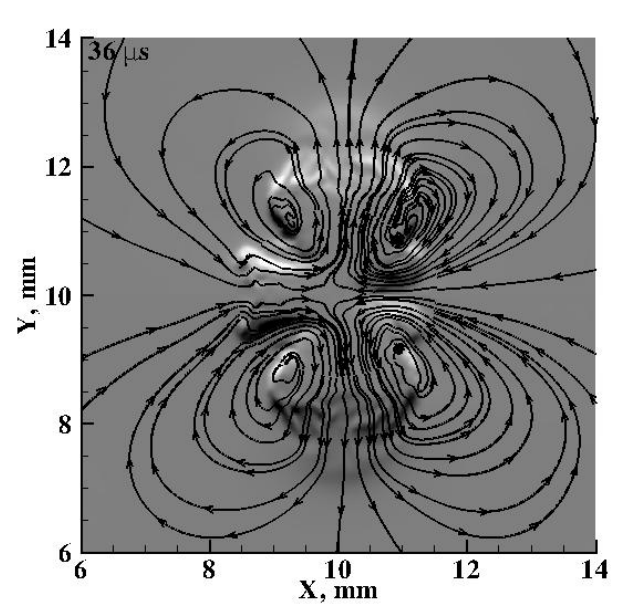

(p)

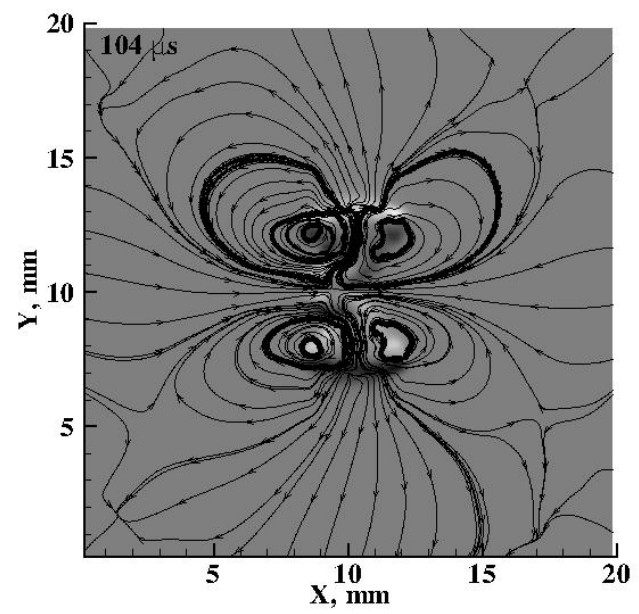

(r) 


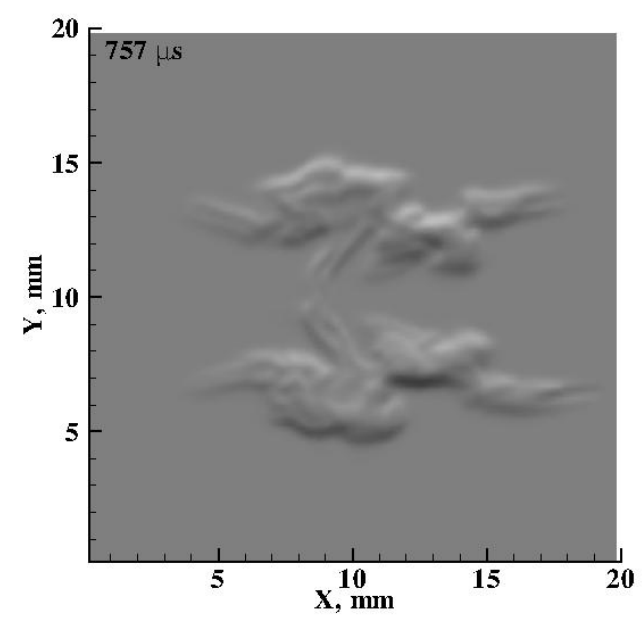

(s)

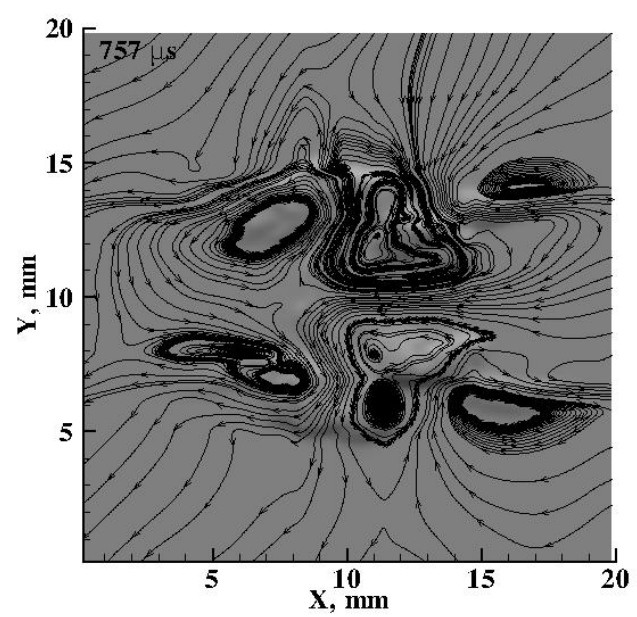

$(\mathrm{t})$

FIG. 9. Streamlines around the central part of the domain at different instants of time. For figures (e-t) only density gradient (along y direction) and streamlines along with density gradient (along y direction) are plotted side by side at the same time instant.

Few authors $[1,4]$ have reported about the appearance of a third lobe in the images of the central part of the domain during later stages of the decay process. The images were obtained by schlieren technique of visualization. The present numerical simulation gives the distribution of density in the domain. A first derivative of the density field in the $\mathrm{Y}$ direction provides numerical schlieren images which are similar to the images obtained by experimental schlieren technique. The numerical schlieren images also show appearance of a third lobe (FIG. 9(m, o)). To understand the reason behind the appearance of the third lobe, streamlines are also plotted side by side (FIG. 9(n, p)). Initially (up to about $0.61 \mu \mathrm{s}$ ) the hot core region and the blast wave expand together and the streamlines change its nature as explained in the previous paragraph. However, after this the blast wave separates itself from the hot core region and the streamline pattern begins to show roll up motion (FIG. 9(j)). The vortices thus generated are not equal in strength and it depends on the initial energy distribution and physicochemical properties of the medium. The roll up motion changes the shape of the core region. For example, if the shape of the core region after detachment of the blast wave is assumed to be ellipsoid with major axis along the $\mathrm{X}$ direction, the roll up motion changes the major axis to $\mathrm{Y}$ direction. However, due differences in strength of the vortices, the ellipsoid nature is lost and it gives the impression of appearance of a third lobe. Bradley [1] assumes that there is a movement of hot gas to the left along the $\mathrm{X}$ axis during this period and hence the third lobe appears. The plot of streamlines from the present 
numerical simulation however, shows that the fluid motion is to the right through the third lobe.

\section{Experimental validation of the blast wave expansion}

Schlieren imaging of blast waves accompanying laser sparks was performed in a laboratory test rig to track its propagation in static air. The experimental setup is shown in FIG. 10. The test rig was a vertically arranged channel with a length of one meter and a square crosssection of $62 \mathrm{~cm}^{2}$. It contained static air at laboratory climate conditions (296 K temperature, $979 \mathrm{hPa}$ static pressure and $29 \%$ humidity). Three of four sides of the channel were equipped with large windows, to enable laser sparking and optical measurement techniques.

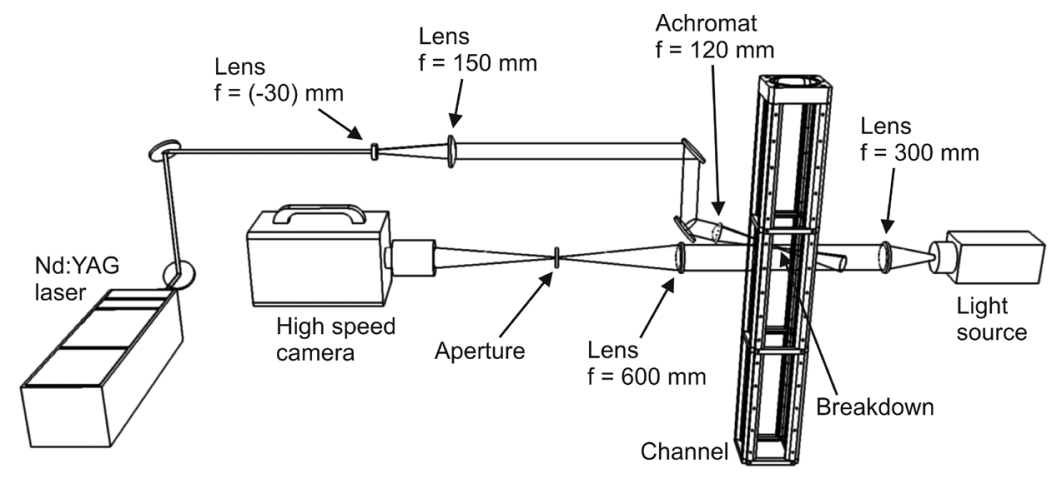

FIG. 10. Experimental setup of the schlieren imaging on laser-induced breakdowns

A frequency-doubled and Q-switched Nd:YAG laser (InnoLas Spitlight 600-10) was used to create the sparks. In a single pulse mode it provided $6 \mathrm{~ns}$ pulses of up to $350 \mathrm{~mJ}$ at $532 \mathrm{~nm}$ and a triggering accuracy of $10 \mathrm{~ns}$. The pulse energy was adjusted through the Pockels cell delay time. Sparks were achieved by expanding the laser beam to a diameter of approximately $40 \mathrm{~mm}$ and refocusing it via a laser-grade achromatic doublet lens with a focal length of $120 \mathrm{~mm}$. The laser beam entered the flow channel with a downward angle of 25 degrees. Part of the laser pulse energy was lost in the optical setup; another part was transmitted through the breakdown region. Therefore, the laser pulse energies directly in front of and behind the breakdown position were measured with a laser power volume absorber (Gentec-EO UP19K-15S-VR-D0). The average pulse energy for 2000 laser shots in front of the breakdown location was $104.9 \mathrm{~mJ}(\mathrm{~s}=0.61 \mathrm{~mJ})$ and $15.2 \mathrm{~mJ}(\mathrm{~s}=0.44 \mathrm{~mJ})$ behind. Consequently, in average $89.7 \mathrm{~mJ}(\mathrm{~s}=0.75 \mathrm{~mJ}$ ) of pulse energy were absorbed in the breakdown. 
A high speed CMOS camera (LaVision HighSpeedStar 6) and a light source (Spindler \& Hoyer lamp) were the main components of the schlieren setup. The beam of the light source was parallelized by a converging lens to a diameter of approximately $100 \mathrm{~mm}$. It passed through the measurement area inside the channel and was refocused by a converging lens on the opposite side. An aperture was placed at the focal point, blocking its lower part in order to generate the schlieren image. The camera was equipped with a Nikkor $180 \mathrm{~mm} \mathrm{f/2.8} \mathrm{lens.} \mathrm{A}$ Schott OG570 filter in front of the lens reduced chromatic aberrations. The camera was operated at a repetition rate of $25 \mathrm{kHz}$, an exposure time of $1 \mu \mathrm{s}$ and a resolution of $448 \times 448$ pixels. The field-of-view was 52 x $52 \mathrm{~mm}$. To achieve a high temporal resolution, the laserinduced breakdown was repeated various times, and the delay between laser pulse and the starting time of the camera exposure was continuously increased by small steps of 0.1 to $2 \mu \mathrm{s}$. Three breakdowns were recorded for every selected delay to account for statistical spread. FIG. 11 shows selected background-corrected schlieren images. The black area in the upperright corner is the bracket of the achromatic lens. The origin of the coordinate axes is the focal point. The white spot in the center comes from the breakdown radiation, which is visible for $17 \mu \mathrm{s}$. The spherical blast wave is apparent in form of a ring of a circular shape, which expands quickly over time. A region of compressed air remains in the center after the breakdown decayed, which results from the fast expansion of the breakdown during the first $2 \mu$ s. It is very stable and mixes with the surrounding air on a timescale of several hundred microseconds.

The radii of the blast waves were derived from the schlieren images with a MATLAB algorithm. The experimental data represents an average over the three data sets for which the analysis was performed. The standard deviation between the radii of the three data sets was below $0.27 \mathrm{~mm}$ with an average of $0.068 \mathrm{~mm}$. 

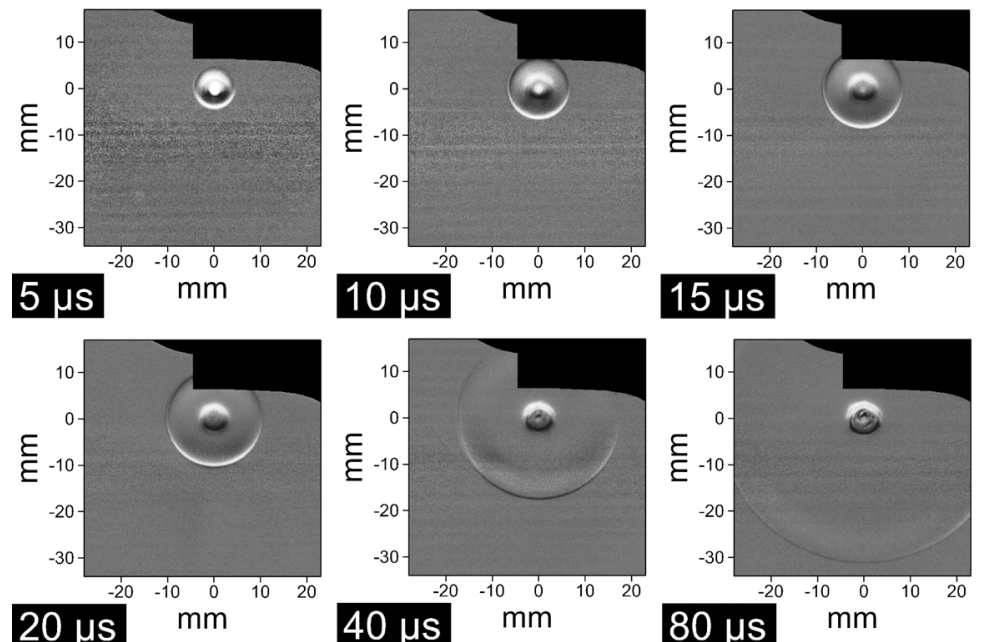

$20 \mu \mathrm{s}$ 40 us

$80 \mu \mathrm{s}$

FIG. 11. Schlieren images of the laser-induced breakdown in static air

The blast wave radius with time from numerical simulation is plotted in FIG. 12. Initially, there are slight differences in the blast wave radius along $\mathrm{x}$ and $\mathrm{y}$ direction as shown in FIG. 12(a). However, those eventually coincide as the blast wave shape becomes circular. In FIG. 12(b), the blast wave radius obtained from numerical simulation is plotted against experimental data. In the early stage, experimental data are not available since a laser spark generates intense light which illuminates the blast wave. Beyond $19.3 \mu \mathrm{s}$, simulation data is not available because the blast wave goes out of the simulation domain. Within the overlapped time, the results are in good agreement.
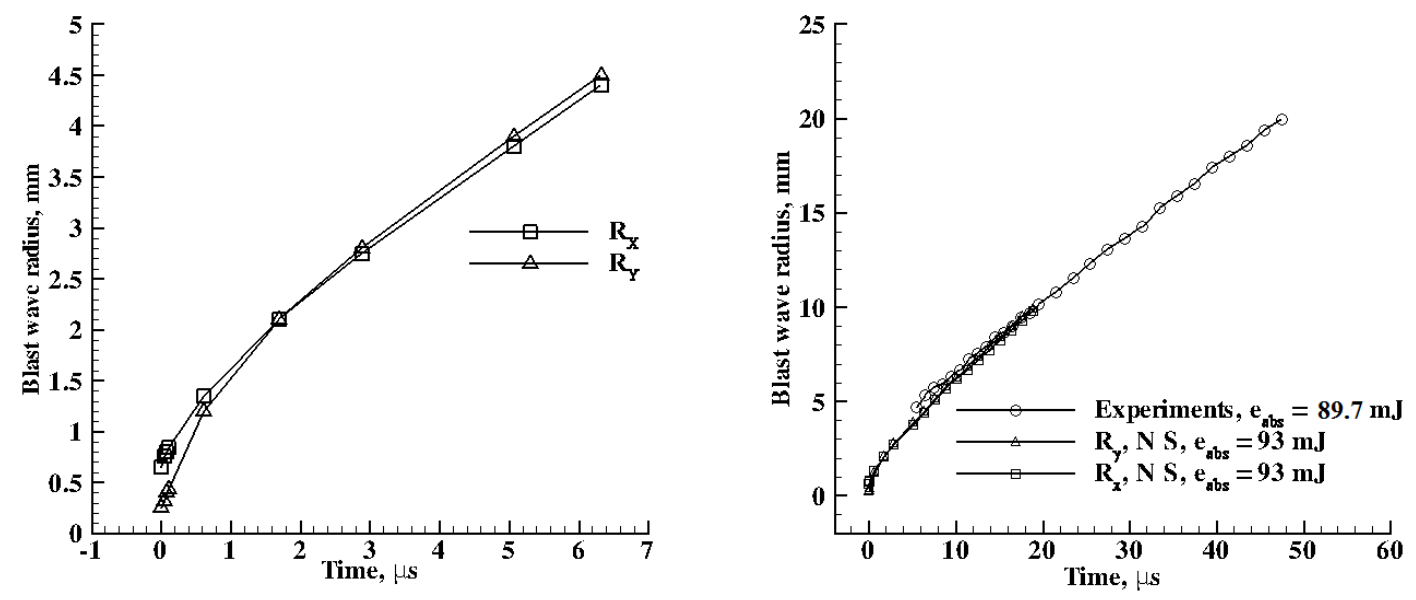

FIG. 12. (a) The blast wave radius along $\mathrm{x}$ and $\mathrm{y}$ directions at different instants of time obtained from numerical simulation, (b) Blast wave radius from experiments and numerical simulation superimposed.

\section{Energy calculation}


The total energy of a fluid dynamic system consists of internal energy and kinetic energy. In the present simulation, the calculations start with an assumed internal energy per unit volume (absorbed energy) and zero kinetic energy. However, during the evolution process, part of the internal energy is converted into blast wave energy and radiation heat energy.

As described in section IV, the blast waves moves quickly in radially outward direction leaving a region containing dissociated species around the center of the domain. The blast wave does not support the ignition process of combustible gases which is observed experimentally by Phuoc et al. [28]. The reason could be that the blast wave carries little internal energy with it and hence the temperature is not sufficient to initiate ignition in a combustible gas. However, the blast wave induces fluid velocity behind it and the blast wave energy refers to the kinetic energy of the induced fluid velocity. The blast wave energy is ultimately lost due to viscous dissipation.

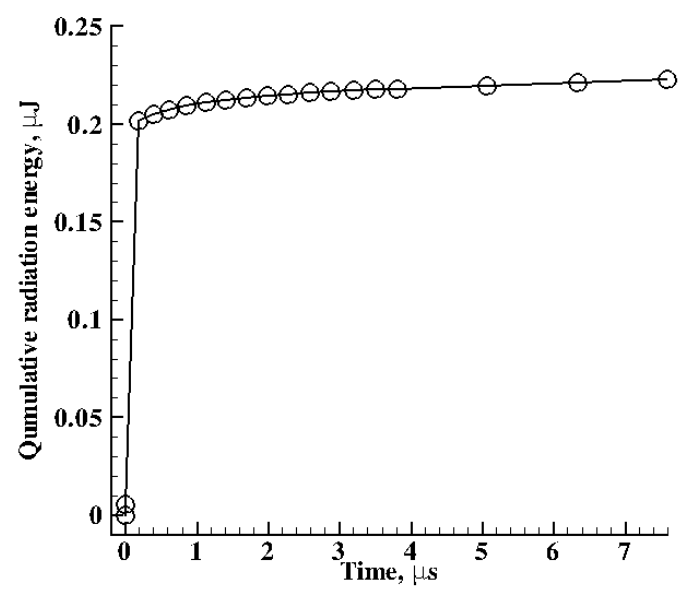

(a)

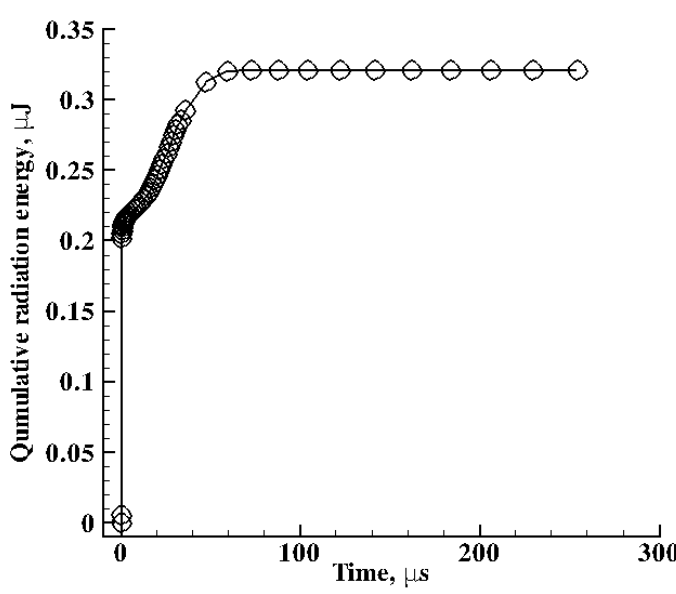

(b)

FIG. 13. Variation of cumulative radiation energy with time (a) during early stages and (b) during the entire decay process.

FIG. 13. shows cumulative radiation heat loss with time. The radiation heat loss is calculated by multiplying the divergence of radiation heat flux term $\nabla . q^{\text {rad }}$ by a square cylinder of volume $2 \pi \mathrm{r} \Delta \mathrm{x} \Delta \mathrm{y}$ and $\Delta \mathrm{t}$ and adding the product for all such volumes for the total solution time. Here $\mathrm{r}$ is the distance of the center of a two-dimensional control volume from the axis of symmetry, $\Delta \mathrm{x}$ and $\Delta \mathrm{y}$ are the length of the sides of a control volume, $\Delta \mathrm{t}$ is the time step for all control volumes. 


$$
\text { Radiation heat loss }=\sum_{t} \sum_{x} \sum_{y} \nabla \cdot q^{r a d}(2 \pi r \Delta x \Delta y \Delta t)
$$

In the calculation of the percentage of total energy left after passage of the blast wave, energy per unit volume is taken as the basis. The profile of energy per unit volume along $\mathrm{x}$ and y direction at several time instants are plotted in FIG. 14. The profiles look like steps. For ease of understanding, the profiles are shown for time instants greater than or equal to 0.39 $\mu \mathrm{s}$. The height of the steps during this time decreases at slower rate than earlier times and because the blast wave carries little internal energy along with it, the energy corresponding to the step height should be able to provide a source of heat and dissociated species. In other words, this energy $\left(1.5 \mathrm{MJ} / \mathrm{m}^{3}\right)$ should be taken as the energy left for ignition. The calculated distribution of total absorbed energy is given in TABLE I.
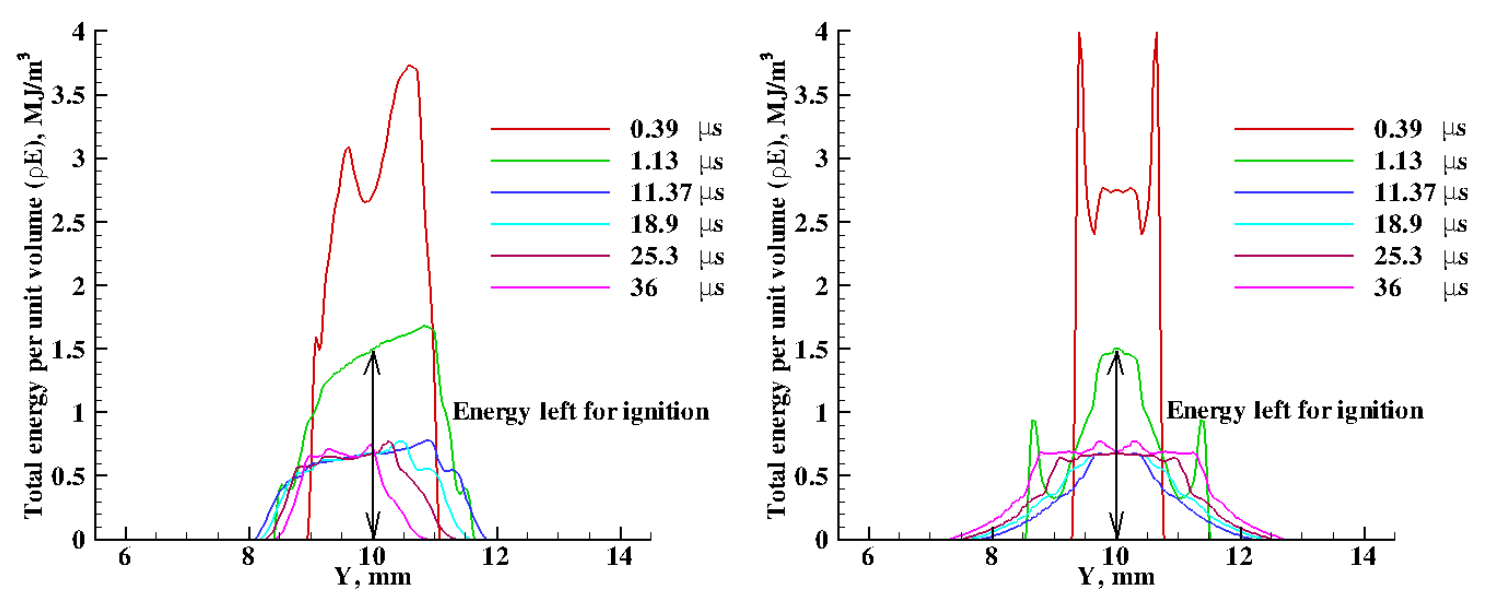

FIG. 14. Line plots of total energy per unit volume along lines passing through the middle of the domain along $\mathrm{x}$ and $\mathrm{y}$ directions during the decay process.

TABLE I. Energy distribution.

\begin{tabular}{ll}
\hline \hline Energy left per unit volume (MJ/m $\left.{ }^{3}\right)$ (from FIG. 14) & 1.5 \\
Energy absorbed per unit volume $\left(\mathrm{MJ} / \mathrm{m}^{3}\right)$ (assumed) & $93 / 3=31$ \\
Percentage of energy left for ignition (\%) & $1.5 / 31=4.84$ \\
Energy left for ignition $(\mathrm{mJ})$ & $93 \times 0.0484=4.5$ \\
Radiation heat loss $(\mu \mathrm{J})$ & 0.33 \\
Blast wave energy $(\%)$ & 95.15 \\
Blast wave energy $(\mathrm{mJ})$ & 88.4 \\
\hline \hline
\end{tabular}




\section{Summary and conclusions}

This paper uses numerical simulation to study the fluid dynamic, physical and chemical changes that arise upon focused laser energy deposition in air. The simulation solves the compressible Navier-Stokes equations along with species conservation equations by the finite volume method. The radiation loss is also modeled by the finite volume method. An assumption is made about the initial distribution of temperature in the laser induced breakdown zone. Local thermodynamic equilibrium conditions are assumed to apply.

The evolution of the decaying laser spark is found to be consisting of two temporal phases. In the first phases, rapid changes in the flow and physical variables occur while, in the second phase changes become much slower and more regular. The evolution of blast wave radius with time is plotted and compared with in-house experimental data. Distinct roll up motion and toroidal ring vortex appear in the second phase of the evolution process. The radiation heat loss is found to be negligible. The remarkable feature observed is the distinct heat transfer mechanisms in the two phases mentioned above. In the first phase convection and radiation are the dominant heat transfer mechanisms while it is conduction in the second phase. The blast wave is found to be carrying little internal energy along with it, leaving behind a region consisting of dissociated species at high temperature but at normal pressure. Based on this, a prediction is made about the actual available energy for ignition of a combustible mixture by a laser spark. The study enhances the understanding of basic flow features and possible ignition energy arising out of a laser induced breakdown process. 


\section{Appendix}

$\mathrm{U}=\left[\begin{array}{l}\rho \\ \rho u \\ \rho v \\ \rho E \\ C_{1} \\ C_{2} \\ \cdot \\ \cdot \\ C_{N-1}\end{array}\right], \mathrm{H}=\left[\begin{array}{l}\rho \vec{V} \\ \rho u \vec{V}+p \vec{I}_{x} \\ \rho v \vec{V}+p \vec{I}_{y} \\ (\rho E+p) \vec{V} \\ C_{1} \vec{V} \\ C_{2} \vec{V} \\ \cdot \\ C_{N-1} \vec{V}\end{array}\right], \mathrm{F}=\left[\begin{array}{l}0 \\ \tau_{x x} \hat{\kappa}_{x}+\tau_{x y} \hat{\kappa}_{y} \\ \tau_{x y} \hat{\kappa}_{x}+\tau_{y y} \hat{\kappa}_{y} \\ \Omega_{x} \hat{\kappa}_{x}+\Omega_{y} \hat{\kappa}_{y}-C_{1} \tilde{u}_{1} \hat{\kappa}_{x}-C_{1} \tilde{v}_{1} \hat{\kappa}_{y} \\ \cdot \\ \cdot \\ -C_{N-1} \tilde{u}_{N-1} \hat{\kappa}_{x}-C_{N-1} \tilde{v}_{N-1} \hat{\kappa}_{y}\end{array}\right], \mathrm{S}=\left[\begin{array}{l}0 \\ 0 \\ 0 \\ 0 \\ S_{1} \\ S_{2} \\ \cdot \\ . \\ S_{N-1}\end{array}\right]$

$S^{1}=\left[\begin{array}{l}0 \\ 0 \\ 0 \\ -\nabla \cdot q^{r a d} \\ 0 \\ 0 \\ \cdot \\ 0\end{array}\right]$

Here the physical variables are the density $\rho$, the velocity components $u, v$ in $\mathrm{x}, \mathrm{y}$ directions respectively, the pressure $p$, the temperature $T$, the internal energy $e$, and the total energy $E=e+\frac{1}{2}\left(u^{2}+v^{2}\right), \quad e=\sum_{i=1}^{N} e_{i} \frac{C_{i}}{\rho W_{i}}, \quad \mathrm{~N}$ is the total number of species, $e_{i}=\int_{T_{R}}^{T} C_{p i} d T+h_{f i}^{0}-R_{u} T$ is the internal energy (molar) of the $i^{\text {th }}$ species, $C_{i}, W_{i}, C_{p i}, h_{f i}^{0}$ are the mass concentration, molecular weight, constant pressure specific heat (molar) and heat of formation of species $i$ respectively, $T_{R}$ is the reference temperature for thermodynamic properties and $R_{u}$ is the universal gas constant. The specific heat of individual species $C_{p i}$ appearing above is calculated by statistical mechanics and fitted in a polynomial of temperature. The polynomial expressions and coefficients could be found in Pimentela et al. [24] and Gupta et al. [25]. $\vec{V}=u \vec{I}_{x}+v \vec{I}_{y} \cdot \vec{I}_{x}, \vec{I}_{y}$ are the unit vectors along $\mathrm{x}, \mathrm{y}$ directions, 


$$
\begin{aligned}
& \tau_{x x}=\mu\left(\frac{4}{3} \frac{\partial u}{\partial x}-\frac{2}{3} \frac{\partial v}{\partial y}\right), \tau_{x y}=\mu\left(\frac{\partial u}{\partial y}+\frac{\partial v}{\partial x}\right), \tau_{y y}=\left(\frac{4}{3} \frac{\partial v}{\partial y}-\frac{2}{3} \frac{\partial u}{\partial x}\right), \Omega_{x}=u \tau_{x x}+v \tau_{x y}+k \frac{\partial T}{\partial x} \\
& -\sum_{i=1}^{N} h_{i} C_{i} \tilde{u}_{i}, \Omega_{y}=u \tau_{x y}+v \tau_{y y}+k \frac{\partial T}{\partial y}-\sum_{i=1}^{N} h_{i} C_{i} \tilde{v}_{i}
\end{aligned}
$$

$\vec{\kappa}, \hat{\kappa}_{x}, \hat{\kappa}_{y}, h_{i}, \tilde{u}_{i}, \tilde{v}_{i}$ are the outward normal to $d s$, components of $\vec{\kappa}$ along $\mathrm{x}$ and y directions, specific enthalpy, diffusion velocities of the species $i$ along $\mathrm{x}$ and $\mathrm{y}$ directions respectively. The diffusion velocities are found from Fick’s law

$$
Y_{i} \tilde{u}_{i}=-D_{i m} \frac{\partial Y_{i}}{\partial x}, Y_{i} \tilde{v}_{i}=-D_{i m} \frac{\partial Y_{i}}{\partial y}
$$

where $Y_{i}=\frac{C_{i}}{\rho}$ and $D_{i m}$ are the mass fraction and effective binary diffusivity of the species $i$ in the gas mixture. The value of $D_{i m}$ is taken as constant and equal to $5 \times 10^{-5} \mathrm{~m}^{2} / \mathrm{s}$ (average value) for all species in the present calculation due to non-availability of data in the open literature for temperature variation of mass diffusivity of the species considered in the calculations. The viscosity and thermal conductivity of individual species are taken as temperature dependent; the temperature dependence is polynomial and could be found in Gupta et al [24].

For a set of $\mathrm{N}_{\mathrm{R}}$ elementary reactions involving $\mathrm{N}$ species the rate equations can be written in the form

$$
\sum_{j=1}^{N} v_{i j}^{\prime} n_{j} \Leftrightarrow \sum_{j=1}^{N} v_{i j}^{\prime \prime} n_{j}
$$

Where $i=1,2, \ldots, \mathrm{N}_{\mathrm{R}}$ is the number of reactions, $v_{i j}^{\prime}$ and $v_{i j}^{\prime \prime}$ are the stoichiometric coefficients for species $\mathrm{j}$ appearing as a reactant in the $i^{\text {th }}$ forward and backward reactions respectively and $n_{j}=\frac{C_{j}}{W_{j}}$ is the molar concentration for species $\mathrm{j}$. The reaction rate constants are given by the Arrhenius expression

$$
k_{i}=A_{i} T^{m i} e^{-E_{i} / R_{u} T}
$$

where $E_{i}$ represents the activation energy of reaction $i$ and $A_{i}, m_{i}$ are constants. $S_{j}$ is obtained by summing up the changes in mass concentrations of species $j$ due to all reactions i.e. 


$$
S_{j}=W_{j} \sum_{i=1}^{N_{R}}\left[\left(v_{i j}^{\prime \prime}-v_{i j}^{\prime}\right)\left(k_{f_{i}} \prod_{l=1}^{N} n_{l}^{v_{i l}^{\prime}}-k_{b_{i}} \prod_{l=1}^{N} n_{l}^{v_{i l}^{\prime \prime}}\right)\right]
$$

Here $k_{f i}, k_{b i}$ are the forward and backward reaction rate constants respectively.

The expressions for the variables appearing in equation 2 are

$$
\begin{aligned}
& \tilde{\alpha}_{1}=\frac{\tilde{a}}{\sqrt{2} \tilde{\rho}}\left(\Delta \rho-\tilde{\alpha}_{3}-\frac{\tilde{\rho} \Delta u_{n}}{\tilde{a}}\right) \\
& \tilde{\alpha}_{2}=\Delta u \tilde{\kappa}_{y}-\Delta v \tilde{\kappa}_{x} \\
& \tilde{\alpha}_{3}=\frac{\tilde{p}_{e}}{\tilde{\rho} \tilde{a}^{2}}\left(\tilde{H} \Delta \rho-\Delta(\rho E)-\frac{\tilde{\rho}^{2}}{\tilde{p}_{e}} \sum_{j=1}^{N-1} \tilde{p}_{C_{j}} \alpha(3+j)+\tilde{\rho}(\tilde{u} \Delta u+\tilde{v} \Delta v)\right. \\
& \tilde{\alpha}_{(4-(N+2))}=\frac{\Delta C_{1-(N-1)}}{\tilde{\rho}}-\left(\frac{\tilde{C}_{1-(N-1)}}{\rho}\right) \frac{\Delta \rho}{\tilde{\rho}} \\
& \alpha_{(N+3)}=\frac{\tilde{a}}{\sqrt{2} \rho}\left(\Delta \rho-\tilde{\alpha}_{3}+\frac{\tilde{\rho} \Delta u_{n}}{\tilde{a}}\right)
\end{aligned}
$$

Here

$$
\begin{gathered}
\tilde{H}=\tilde{h}+\frac{1}{2}\left(\tilde{u}^{2}+\tilde{v}^{2}\right), \tilde{a}^{2}=\frac{\tilde{p}}{\tilde{\rho}}\left(1+\frac{R}{\tilde{C}_{v}}\right), \tilde{u}_{n}=\tilde{u} \tilde{\kappa}_{x}+\tilde{v} \tilde{\kappa}_{y} \\
\Delta(.)=()_{R}-(.)_{L} \\
\tilde{p}_{e}=\bar{p}_{e}\left(1+\frac{\bar{p}_{e} \Delta e}{\left(\bar{p}_{e} \Delta e\right)^{2}+\left(\bar{p}_{\rho} \Delta \rho\right)^{2}+\sum_{i=1}^{N-1}\left(\bar{p}_{C_{i}} \Delta C_{i}\right)^{2}}\right) \delta p \\
\tilde{p}_{\rho}=\bar{p}_{\rho}\left(1+\frac{\left(\bar{p}_{e} \Delta e\right)^{2}+\left(\bar{p}_{\rho} \Delta \rho\right)^{2}+\sum_{i=1}^{N-1}\left(\bar{p}_{C_{i}} \Delta C_{i}\right)^{2}}{\bar{p}_{\rho} \Delta \rho}\right) \delta p \\
\tilde{p}_{C_{i}}=\bar{p}_{C_{i}}\left(1+\frac{\bar{p}_{C_{i}} \Delta C_{i}}{\left(\bar{p}_{e} \Delta e\right)^{2}+\left(\bar{p}_{\rho} \Delta \rho\right)^{2}+\sum_{i=1}^{N-1}\left(\bar{p}_{C_{i}} \Delta C_{i}\right)^{2}}\right) \delta p, i=1,2, . ., N-1
\end{gathered}
$$

where $\bar{p}_{e}, \bar{p}_{\rho}, \bar{p}_{C_{i}}$ are quantities determined at Roe average physical variables and

$$
\delta p=\Delta p-\left(\bar{p}_{\rho} \Delta \rho+\bar{p}_{e} \Delta e+\sum \bar{p}_{C_{i}} \Delta C_{i}\right)
$$



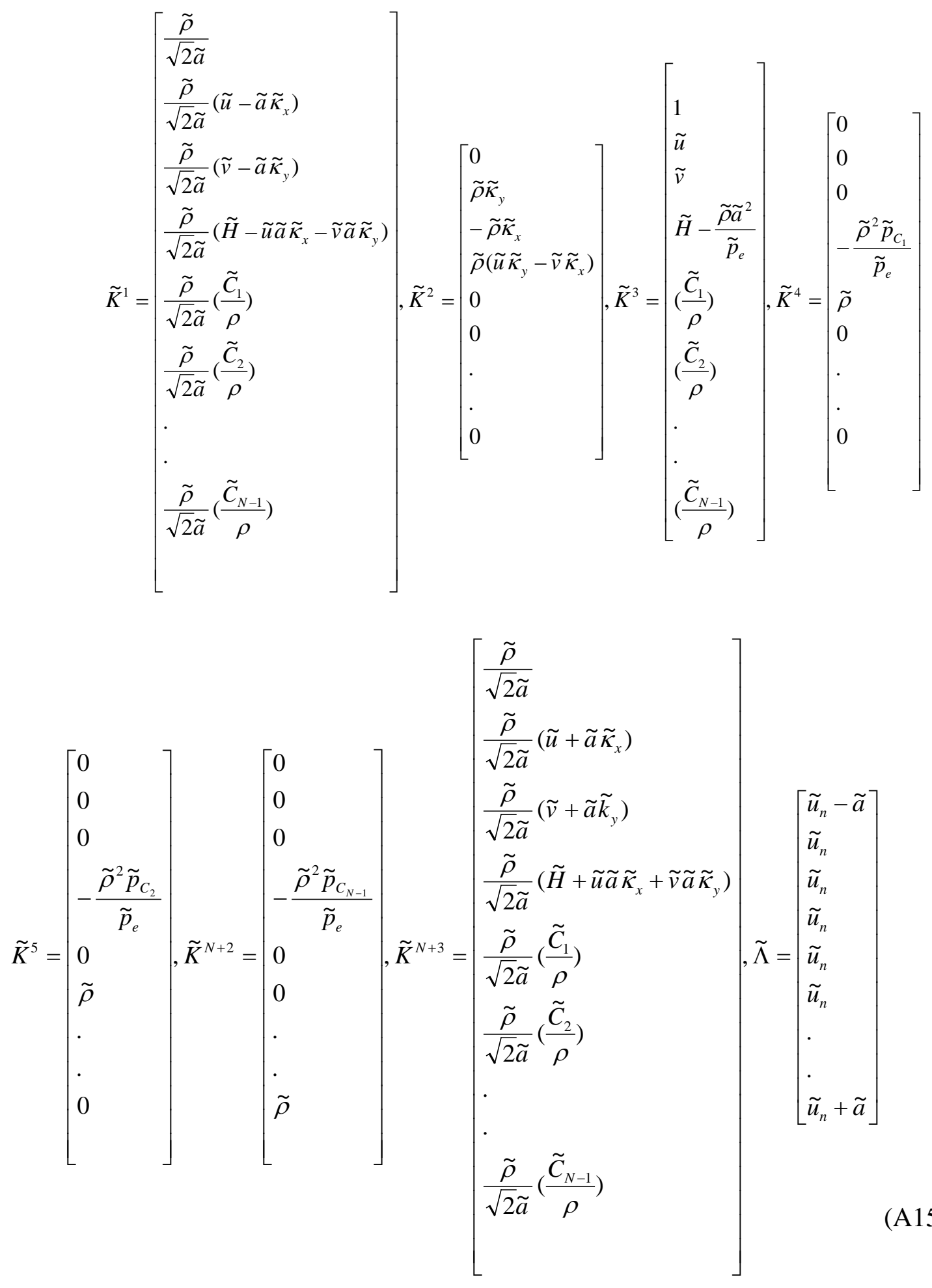


\section{References}

${ }^{1}$ D. Bradley, C.G.W. Sheppard, I.M. Suardjaja, and R. Woolley, “Fundamentals of highenergy spark ignition with lasers Combustion and Flame”, 138(2004), 55-77

${ }^{2}$ Tran X. Phuoc, “'Laser-induced spark ignition fundamental and applications“, Optics and Lasers in Engineering, 44(2006), 351-397

${ }^{3}$ Paul D. Ronney, “'Laser versus conventional ignition of flames“, Optical Engineering 33(2), 510-521 (February 1994)

${ }^{4}$ I. Dors, C. Parigger, and J. Lewis, “Fluid dynamics effects following laser-induced optical breakdown“, AIAA 2000-0717 (2000)

${ }^{5}$ Ramnath Kandala, Graham V. Candler, “Computational Modeling of Localized Laser Energy Deposition in Quiescent Air“, 33rd Plasmadynamics and Lasers Conference, AIAA 2002-2160, 20-23 May 2002, Maui, Hawaii

${ }^{6}$ Ivan G. Dors and Christian G. Parigger, "Computational fluid-dynamic model of laserinduced breakdown in air”, Applied Optics, Vol. 42, No. 30, 2003

${ }^{7}$ H. Yan, R. Adelgren, M. Boguszko, G. Elliott, D. Knight, “'Laser Energy Deposition in Quiescent Air“, AIAA 2003-1051 (2003)

${ }^{8}$ Shankar Ghosh and Krishnan Mahesh, "Numerical simulation of the fluid dynamic effects of laser energy deposition in air“, J. Fluid Mech. (2008), vol. 605, pp. 329-354

${ }^{9}$ J. B. Simeonsson and A. W. Miziolek, "Time-resolved emission studies of ArF-laserproduced microplasmas“, Appl. Opt. 32, 939-947, 1993

${ }^{10}$ Bin Wang, Kimiya Komurasaki, Toshikazu Yamaguchi, Kohei Shimamura, and Yoshihiro Arakawa, “Energy conversion in a glass-laser-induced blast wave in air“, Journal of Applied Physics 108, 124911 (2010) 
${ }^{11}$ P. L. Roe, “Approximate Riemann solvers, parameter vectors, and difference schemes“, Journal of Computational Physics, Vol. 43, 1981, 321-326

${ }^{12}$ Jian-Shun Shuen, "'Upwind differencing and LU factorization for chemical non-equilibrium Navier-Stokes equations“, Journal of Computational Physics, 99:233-250, 1992

${ }^{13}$ E. F. Toro, "Riemann solvers and numerical methods for fluid dynamics: A practical introduction“, Springer-Verlag, Berlin, 1999

${ }^{14} \mathrm{~B}$. Van Leer, "Towards the ultimate conservative difference scheme, V. A second-order sequel to Godunov’s method“, Journal of Computational Physics, 32:101-136, 1979

${ }^{15}$ G. D. Van Albada, B. Van Leer, and W. W. Roberts, "A comparative study of computational methods in cosmic gas dynamics“, Astron. Astrophys., 108:76-84, 1982

${ }^{16}$ R. Peyret and D. T. Taylor, Computational Methods for Fluid Flow, Springer, Berlin, 1983

${ }^{17}$ John C. Chai, HaeOk S. Lee, Suhas V. Patankar, “Finite Volume Method for Radiation Heat Transfer“, Journal of Thermophysics and Heat Transfer, Vol. 8, No. 3, July-Sept. 1994

${ }^{18}$ J. Y. Murthy and S. R. Mathur, "Finite Volume Method for Radiative Heat Transfer Using Unstructured Meshes“, Journal of Thermophysics and Heat Transfer, Vol. 12, No. 3, JulySeptember 1998

${ }^{19} \mathrm{~N}$ Bogatyreva, M Bartlova and V Aubrecht, “Mean absorption coefficients of air plasmas“, 11th European Conference on High-Technology Plasma Processes (HTPP 11), Journal of Physics: Conference Series 275 (2011) 012009, IOP Publishing

${ }^{20}$ Thomas R. A. Bussing and Earll M. Murman, "Finite-volume method for the calculation of compressible chemically reacting flows“, AIAA Journal, 26(5):1070-1078, 1987

${ }^{21}$ K. G. Powell and B. Van Leer, "Tailoring explicit time marching schemes to improve convergence characteristics“, Technical report, VKI LS 1990-03, Computational Fluid Dynamics, March 1990 
${ }^{22}$ Sanford Gordon and Bonnie J. McBride, “Computer program for calculation of complex chemical equilibrium composition and applications“, NASA Reference Publication, 1311, October, 1994

${ }^{23}$ J. S. Shuen, M. S. Liou, and B. V. Leer, “'Inviscid flux-splitting algorithms for real gases with non-equilibrium chemistry“, Journal of Computational Physics 90, 371-395, 1990

${ }^{24}$ Carlos Alberto Rocha Pimentela and Annibal Hetem Jr., “Computation of air chemical equilibrium composition until 30000K - Part I“, J. Aerosp. Technol. Manag., São José dos Campos, Vol.3, No.2, pp. 111-126, May-Aug., 2011

${ }^{25}$ Roop N. Gupta, Jerrold M. Yos, Richard A. Thompson, and Kam-Pui Lee, “A Review of Reaction Rates and Thermodynamic and Transport Properties for an 11-Species Air Model for Chemical and Thermal Nonequilibrium Calculations to $30000 \mathrm{~K}$ “, NASA Reference Publication 1232, 1990

${ }^{26}$ Adelgren, R. G., Yan, H., Elliott, G. S., Knight, D., Beutner, T. J., Zheltovodov, A., Ivanov, M. \& Khotyanovsky, D., “Localized flow control by laser energy deposition applied to Edney IV shock impingement and intersecting shocks“, AIAA Paper 2003-31 (2003)

${ }^{27}$ Glumac, N., Elliott, G. \& Boguszko, M., “'Temporal and spatial evolution of the thermal structure of a laser spark in air“, AIAA Paper 2005-0204 (2005)

${ }^{28}$ Tran X. Phuoc and Fredrick P. White, “An optical and spectroscopic study of laser-induced sparks to determine available ignition energy“, Proceedings of the Combustion Institute, Volume 29, 2002, pp. 1621-1628 